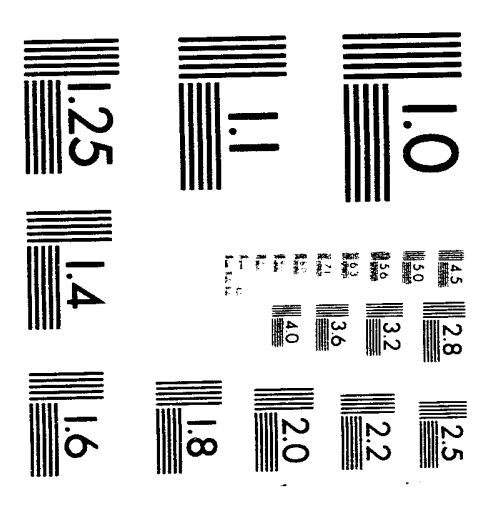



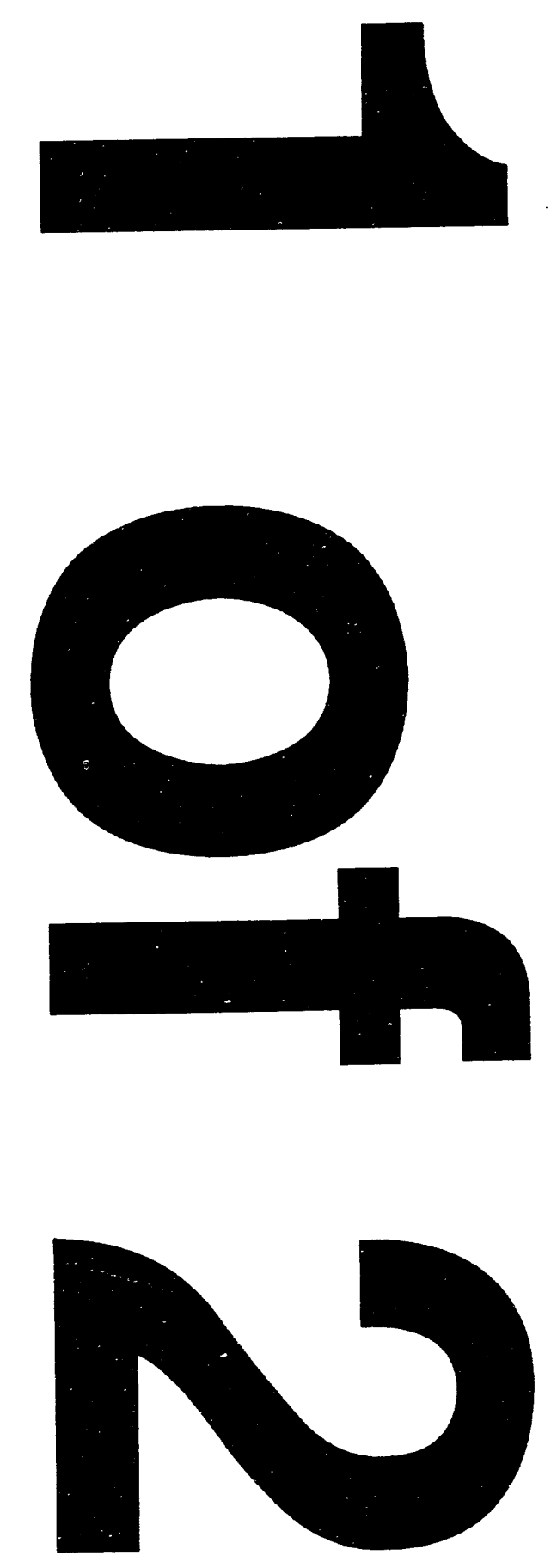


\title{
Gammasphere Software Development
}

\author{
Progress Report
}

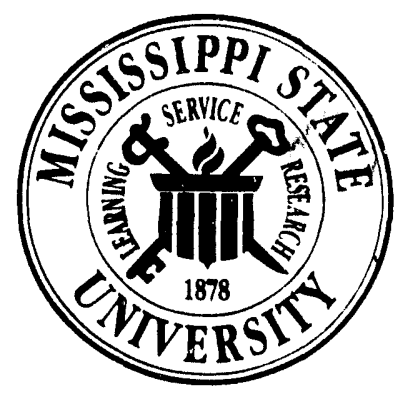

Department of Physics and Astronomy

Mississippi State University

Mississippi State, Mississippi 39762

Prepared for the U.S. Department of Energy

Under Contract Number DE-FG05-92ER40726 


\title{
Gammasphere Software Development
}

\author{
Progress Report
}

Department of Physics and Astronomy

Mississippi State University

Mississippi State, Mississippi 39762

Prepared for the U.S. Departiment of Energy

Under Contract Number DE-FG05-92ER40726
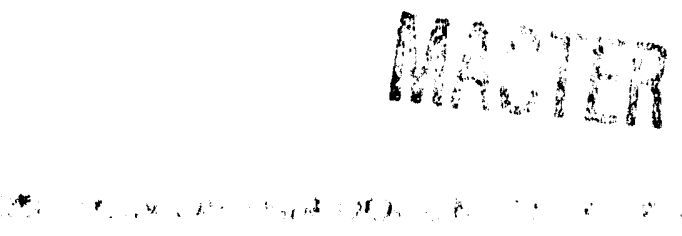

$\pi^{\beta}$ 
Gammasphere Software Development

Rodney B. Piercey

Associate Professor of Physics

Mississippi State University

January, 1994 


\section{Contents}

Summary

I. Introduction

II. Student Involvement

1. Graduate Students

2. Undergraduate Students

3. High School Students

III. Activities of the Software Working Group

IV. Development of a Histogram Format

1. The Universal Histogram Object

2. The SHOW application Example

V. Development of a User Interface Shell

VI. An FTP Site for Software Development Support

VII. Testing the ORNL SUNPAK Software

VIII. Other Work

IX. Publications, Abstracts and Presentations

X. Project Chronology

Appendix 1. UHO C Language Header Files

Appendix 2. Preliminary SUNPAK Test Results

Appendix 3. MSU FTP Site Manual 


\section{Summary}

This report describes the activities of the nuclear physics group at Mississippi State University which were performed during the 1993 calendar year under contract DE-FG0592ER40726 with the Department of Energy. During 1993, the second year of our project, we have made significant progress in our focus areas: chairing the Gammasphere Software Working Group (SWG); assisting with the porting and enhancement of the ORNL UPAK histograrnming software package; and developing standard formats for Gammasphere data products. In addition, we have established an new public ftp archive to distribute software and software development tools and information.

We are continuing to provide leadership for the Gammasphere Software Working Group (SWG) by organizing and chairing meetings, distributing Working Group summaries and disseminating related information. The SWG had its third full meeting during this grant period and three new members were added. The meeting summary has been compiled and distributed to interested members of the Nuclear Physics Community. Additional copies are available on request. A complete report was provided to the Gammasphere User's Group at the APS Division of Nuclear Physics meeting. A summary of the report was included in the most recent Gammasphere News Letter. The three standing subcommittees also continue to make valuaile contributions in their specific areas.

The subcommittee on a "standard format for histogram data" has developed a semi-final version of the Universal Histogram Object (UHO) specification. The basic specification has been reported at two professional meetings and input from numerous researchers has been considered. A number of developers, including the LBL and ORNL groups, have agreed to include support for the UHO in their histogramming software.

We are continuing to assist software developers in adapting and testing the ORNL SUNPAK software package for use at Gammasphere. We are using the SUNPAK on different Sparcstation configurations with different monitors and with different window managers (OpenLook and Motif). We provide our test results to the software developers at ORNL who are porting and maintaining the SUNPAK software.

Our nuclear group continues to involve several graduate, undergraduate and high school students. Two graduate students and an undergraduate student are partially funded by the project. Mr. Pete Varmette finished his masters degree during this last grant period and is now working on a Ph.D. degree in our group. A very exciting element of our research effort is the involvement of 
high school students from the Mississippi School of Math and Science. Our nuclear research program annually provides a unique experience for the student who participate. 


\section{Introduction}

This report describes the 1993 activities of the nuclear physics research group at Mississippi State University under contract DE-FG05-92ER40726 with the Department of Energy. This document and the accompanying SWG-III summary are submitted to comply with DoE grant requirements. Chapters II-VIII describe the various activities of our research group. Chapter II describes the role of students in our research program. The final two sections of this report list the abstracts, publications and presentations which resulted from our research and a short chronology of relevant events. Also included with this report is the Summary and Presentations of the Third Gammasphere Software Issues Workshop.

\section{Student Involvement}

The MSU nuclear research group recognizes the importance of providing students with a rewarding research experience. Each year we include graduate students, undergraduate students, and high school students in our research program. Two of our graduate students and one of our undergraduates receives partial support from our current grant. One graduate student has already received his masters degree based on research in this program.

\section{Graduate Students}

There are currently four graduate students working in the nuclear physics group at Mississippi State University. Two of these students are working on Gammasphere software development. Mr. Pete Varmette (who spent the summer of 1992 working at LBL with Gammasphere researchers in developing an automated liquid nitrogen fill system for Gammasphere) is continuing to work in several software related areas. These include the development of data formats and testing of the UPAK software from ORNL. Mr. Pete Varmette finished his masters degree in 1993 and is now working on a Ph.D. degree in our group. Ms. Anita Triveda is working primarily in the area of UPAK testing but also works on the development of the SHOW application. She is also part time at the Mississippi State University Engineering Research Center and acts as our liaison there. Two other graduate students, Mr. Morris Fields and Mr. Hazem Tliemat are working in nuclear spectroscopy and relativistic hydrodynamics.

\section{Undergraduate Students}

Two undergraduate students, Mr. Charles Beyer and Mr. Larry Gariepy currently work in the nuclear research group. Mr. Beyer is seeking a B.S. degree in physics and works on UHO 
testing and development. Mr. Gariepy is seeking a double major in Computer Science and Mathematics. His research involves the study of message passing techniques in Solaris, the SUN operating system.

\section{High School Students}

The Nuclear group provides a unique opportunity for local high school students to participate in DoE sponsored research. In a cooperative program between Mississippi State University (MSU) and the Mississippi School for Math and Science (MSMS) in Columbus MS our research group is reaching out to the best, most promising, high school students in Mississippi. Each year the MSMS sends students to MSU to interact with our research group and become involved in hands-on scientific research. The students spend three hours a week doing research at MSU with our group for the entire academic year. At the end of the year each student writes a report and makes a presentation to other students from the MSMS.

This year two MSMS students, Kohl Gill and Drew Pruett, have been applying nuclear spectroscopy techniques experimental data on Gold 186 and Palladium 106. In their research the students have become familiar with our UNIX based computer facility and several analysis programs. They have acquired substantial experience using the Display, Analysis, and Manipulation Module (DAMM). DAMM is part of SUNPAK, a computer software package that was written at Oak Ridge National laboratory and is being supplied to the Gammasphere facility. 


\section{Activities of the Software Working Group}

The Gammasphere Software Working Group (SWG) was established by the director of the Gammasphere project to "generate software that will be useful in Gammasphere operation, data acquisition, and/or data analysis and to interact with Gammasphere management to develop an effective interface for user access to and use of the Gammasphere computer facilities". The SWG grew from nine to thirteen permanent members during this last year. The committee had its third major meeting this year at Lawrence Berkeley Laboratory. The first two SWG meetings were held at Mississippi State University and at the APS Nuclear Physics Division meeting in Santa Fe New Mexico.

The three standing SWG subcommittees continue to contribute in our focus areas. The subcommittee on Data Formats has prepared a semi-final draft of the UHO specification. Adoption of the UHO will encourage data sharing and tool sharing. The draft specification is included in this document as an appendix. The subcommittee on Parallel Programming is tracing developments in distributed parallel systems which could impact the use of the Gammasphere computer system. The subcommittee on Graphical User Interface issues is aiding in the port of the ORNL software to a distributed SUN-based, OpenLook/Motif compatible package. The bound proceedings of the three SWG meetings and the draft UHO specification is available from the SWG committee. 


\title{
IV. Development of a Histogram Format
}

One of the goals of the SWG was to encourage the nuclear physics community to contribute to the development of high quality software for the Gammasphere. The absence of a standard data format was clearly identified as one of the primary obstacles to sharing the software that already existed and the development of new software tools. To study this situation and to develop a data format that could serve the community the subcommittee for a histogram standard was formed. With the help of this committee and others in the nuclear physics community, we have developed a universal histogram objec ${ }^{+}$(UHO) that defines a file format and provides for an object oriented programming model. We are also developing an example application, SHOW, that provides a library of callable subroutines which illustrate basic UHO operations. The UHO header files and the source code for the SHOW program are available on the MSU nuclear group public ftp archive.

\section{The Universal Histogram Object}

The following is a preprint on the UHO file specification:

\section{UHO File format Specification}

\author{
A Universal Histogram Object For Nuclear Science \\ R.B. Piercey, P. Varmette, C.J. Beyer, Mississippi State University \\ R.A. Belshe, Lawrence Berkeley Laboratory \\ R.W. MacLeod, AECL Research \\ and \\ R.L. Varner, Oak Ridge National Laboratory,
}

\begin{abstract}
One of the primary obstacles to widespread sharing of data and data display/analysis tools in nuclear research is the absence of a generally accepted histogram file format. We have developed a universal histogram object (UHO) that defines a file format and provides for an object
\end{abstract}


oriented programming model. The UHO file format consists of a list of self describing records. Each record is identified by a tag and contains the record size. In addition to the data record itself, special record types specify such things as display characteristics, calibrations, annotations, regions, markers, sort conditions, etc. Records and record fields are provided to support graphical user interfaces and heterogeneous distributed systems. The UHO also supports event-by-event data acquisition by providing for "intelligent" histogram objects that can accept event record buffers and sort the events based on Condition and Region records.

\section{Introduction}

In recent years, nuclear and high-energy, experimental research efforts have involved an increasing number of researchers and institutions. The researchers generally share the experimental data from an experiment which may be analyzed by several different groups at their home institutiions. The results of the analysis must then be formated and often redistributed for reveiw and inclusion in reports and manuscripts. Formats for handling and storing the data have been independently developed at several national laboratory and university research sites, but no generally accepted standard has emerged. The existence of many different data formats makes sharing data unnecessarily complex and time consuming. Translation of histogram data often results in duplicated effort. In addition, many of the formats that have developed over the years are platform dependent and do not support heterogeneous network environments. Also, there is little built-insupport for the new GUI and "workspace/workgroup" paridigms.

Several of the heavy-ion laboratories in the US and at least one commercial firm have histogram formats that have been adopted in the user communty. CERN also has distributed a system for displaying and analyzing histogram data, but the histogram data format has not found a wider acceptance than the PAW and HBOOK user communities. The NCSC has created the HDF which provides a very general data format. The HDF system includes formats for histogram data but the format is complex and has not achieved widespread use in the nuclear physics research community.

A widely used, standard format would save many hours of research time and promote data and tool sharing. We have developed a universal histogram object (UHO) which incorporates 
characteristics of formats used at the major research laboratories. The UHO is self describing, platform independent and supports GUI and workspace application models. The UHO is based on object oriented programming concepts and provides both a file format and a programming model. It may be extended by the user to include any application specific requirements.

\section{A Universal Histogram Object}

The Universal Histogram Object (UHO) is a self describing data format that can be used to represent histogram and associated ciata on file media, in cpu memory or during network transmissions. In addition, the UHO suggests an object oriented programming model which includes mechanisms for display, manipulation and analysis tools.

Multi-dimensional histograms of arbitrary size are supported in the UHO. The UHO also provides for associated data structures such as markers, cursors, regions, axes, calibrations, scale factors, etc. These structures can be manipulated interactively by the user and are saved with the histogram data. This insures that when a histogram, which has been saved, is reopened, the "state" of the associated structures can be restored, preserving the users "workspace". Such behavior obviates the necessity of re-formatting the data when it is reopened. By adding new UHO associated structures, the UHO behavioral model may easily be customized and extended by the user.

\section{UHO Design Guidlines}

In designing a format for histogram data, several criteria were applied.

-Usability

The UHO specification provides for suport of graphical user iterfaces and the workspace concept. It is simple to understand and to use. It may be implementated partially or fully depending on the users needs. The programming model can be implemented in such a way that the file format and memory images are (longword) isomorphic.

-Portability

The UHO format is designed to be machine portable. This means that UHO data and formatting information must be readable with many different machine types. In support 
of this requirement, UHO record types are selfidentifying and data fields are 32 bit word aligned. Also bit level representations are discouraged.

- Flexibility

The UHO may be implemented to varying degrees. It may serve only as an intermediate porting format when data is to be moved from one system to another or it may be implemented fully to support file storage, complexGUIs, work space preservation, data analysis, etc. The UHO provides for histogramming in arbitrary dimensions, the definition of regions and conditions, and for event-by-event sorting.

- Extensibility

By allowing additional record types to be defined, the UHO can be extended to support custom applications or new data types.

\section{UHO File Format}

The UHO file is a list of self-identifing elements or records. Each record has a type and a size. This allows an application program to check for record types that it knows and skip those that it does not need or recognize. For example, a application tool which analyzes data in a "batch" mode may not need any information about how to display the data. However, a plotting or drawing program would need detailed information of this type. Each record may also be owned by a parent record so that hierarchical structures are possible.

A list of UHO record types is given in table 1. The "DATA" record contains the actual histogram data and information on the byte order, word size and data lengths. The "ERRS" record contains errors for the data in the "DATA" record and allows errors to be propagated through histogram manipulations and for error bars (or other representations) to be drawn with the data. The "DSPL" record contains the information required to draw the "counts" axis of the histogram. This includes the minimum and maximum values to display and symbol and color information. The "PARM" record includes similar informtion about the channel axes. There will usually be a "PARM" record for each of the dimensions of the histogram. "CALB" records provide calibration information for each of the channel axes. The "MARK" and "REGN" records define markers and regions in the histogram. There may be several of each of these in a UHO object. The "COND" record is a set of conditions or filters which regulate how blocks of events are sorted. A condition 
requires that a particular parameter set be contained in one or more histogram regions. The "ANOT" records are used to add anotations to any of the UHO records.

\begin{tabular}{|l|c|c|l|l|}
\hline \multicolumn{1}{|c|}{ TYPE } & TAG & $\begin{array}{c}\text { Versio } \\
\mathbf{n}\end{array}$ & ST ATUS & Notes \\
\hline Histogram Data & DATA & 1.0 & Develop & \\
\hline Errors Data & ERRS & 1.0 Beta & Develop & $\begin{array}{l}\text { Contains associated } \\
\text { errors data }\end{array}$ \\
\hline Display Info & DSPL & 1.0 & Develop & \\
\hline Parameter Info & PARM & 1.0 & Develop & \\
\hline Calibration Info & CALB & 1.0 Beta & Develop. & \\
\hline Region Info & REGN & 1.0 Beta & Develop & \\
\hline Marker Info & MARK & 1.0 Beta & Develop & \\
\hline Anotation Info & ANOT & 1.0 Beta & Develop & \\
\hline Archive Dir & ADIR & 1.0 Beta & Develop & \\
\hline Archive Data & ARCH & 1.0 Beta & Develop & \\
\hline Animation Data & ANIM & 1.0 Beta & Develop & \\
\hline Frame Data & FRAM & 1.0 Beta & Develop & \\
\hline & & & & \\
\hline & & & & \\
\hline
\end{tabular}

Table 1. The UHO record types.

\section{UHO Record Format}

UHO record types have a common format. Each has a 4-byte tagword in the first 4 bytes that identifies that record type. The total record size is given in the next 4 bytes. A record version and ID are provided to identify and differentiate records of the same base type. The record Owner ID allows records to be associated in a hierarchical fashion. A variable amount of record data can be included since the record size is imbedded in the structure. In order to support file- 
image/memory-image isomorphism on an arbitrary machine, all data are aligned on 32 bit word boundries.

\begin{tabular}{|c|c|l|}
\hline Field & Type & Notes \\
\hline Tag & 4 bytes & \\
\hline Size & size $\_$ & \\
\hline Version & 4 bytes & \\
\hline ID & 4 bytes & \\
\hline Owner ID & 4 bytes & \\
\hline User Data & 4 bytes & \\
\hline Record Data & & \\
$\cdot$ & & \\
$\cdot$ & & \\
$\cdot$ & & \\
\hline
\end{tabular}

Table 2. The UHO record format.

\section{UHO object behavior}

In addition to a file format, the UHO suggest an object oriented model for applications that manipulate the UHO files. A unique feature of the UHO is the conceptual model shown in figure 1 that is suggested for the process of sorting or histogramming multiparameter data. In the UHO model, events or blocks of events are sent to histograms as high-level messages. Each histogram object may have sorting conditions built in via COND records, while the sorting mechanisms are included as UHO member functions or methods. In an object oriented implementation, the sort conditions could be configured interactively by the user and stored as a part of the histogram object. 


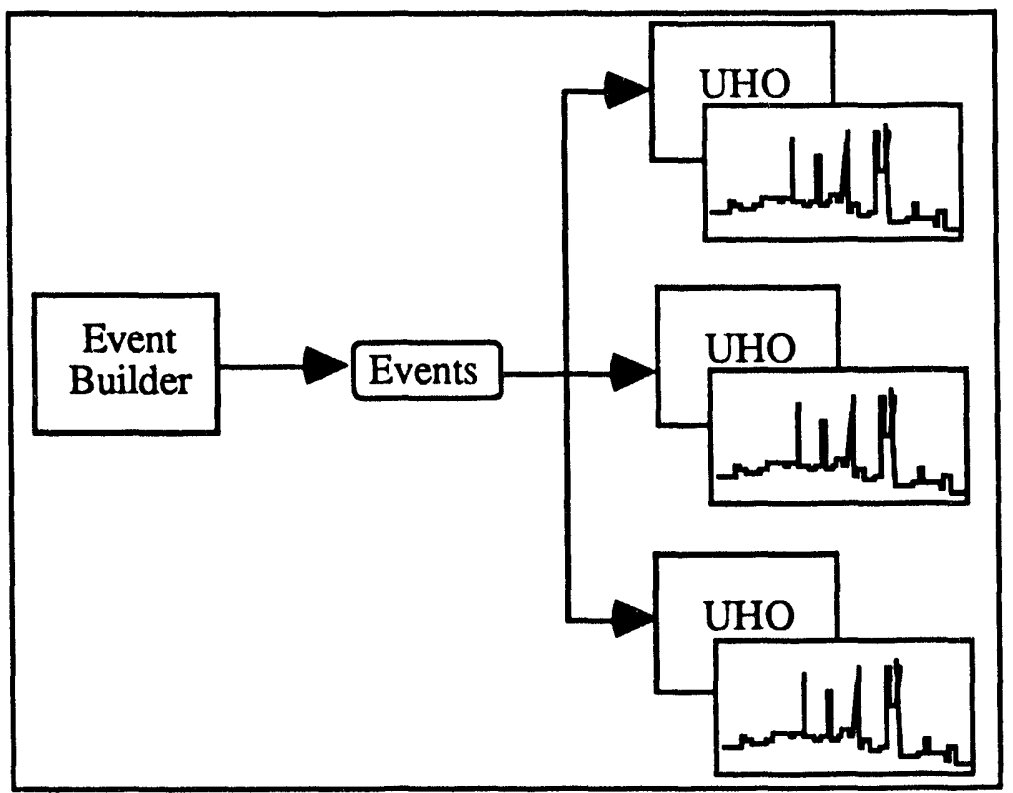

Figure 1. Event messages containing the multi-parameter event data are sent to the UHO which contains the sort conditions as member data and the sorting procedures as member functions. Other member functions are called to display and manipulate the histogram. 


\section{The SHOW application Example}

\section{SHOW: A UHO Example Implementation}

We have developed an example application (SHOW) which illustrates the use of most of the UHO records and suggests a rudimentary interface for manipulating the record objects. The Show program is able to read, write and display UHO files and provides a graphical user interface for manipulating certain characteristics of the data.

\section{Show Memory Management}

While both static and dynamic memory models may be used to manipulate UHO files, in general an application tool or program that intends to read a UHO file into memory will not know in advance how much memory is required. In these cases memory must be allocated dynamically. The Show application uses a dynamic memory model and maintains the various UHO records as a number of linked lists or queues. Fields in each record are reserved to store the pointer to the next item in the list. A structure is allocated to store the pointers to the first element in each list. and each list item will then point to the next list element as shown in figure 2 . List items may be added or removed by user interactions or other processes. In such interactive models, memory may often become fragmented and unusable as hundreds of list elements are allocated and de-allocated. Depending on the OS memory management sophistication, the programmer may be required to perform periodic maintainance to insure an efficient memory map of the associated items in the list.

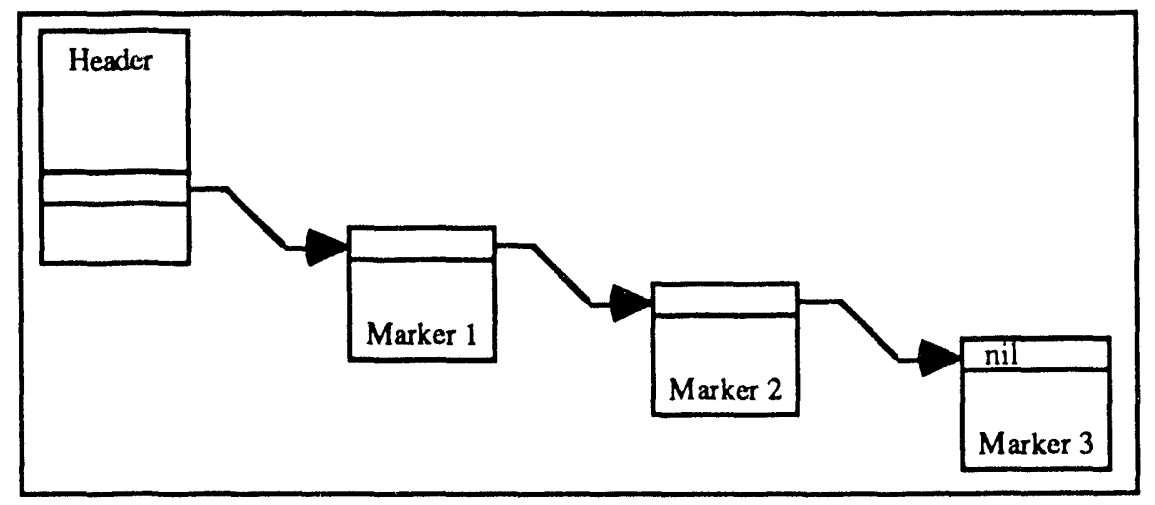

Figure 2. UHO records of a given type are maintained as linked lists in memory.

The current version of SHOW is implemented in C, a procedural programming language. The Show application provide procedures to allocate, de-allocate and manipulate each linked list. In a full implementation of the UHO model, object oriented programming may be used to 
encapsulate the UHO data and member functions. When the histogram is saved or closed, the disk file is updated to reflect the new list elements. The separation of algorithmic and user-interface code in the SHOW example is consistent with client-server models and distributed systems. In general the UHO/SHOW model may be implemented in a wide variety of hardware and software environments.

\section{Conclusions}

In the current prototype implementation, SHOW, the UHO model has proven to be flexible and powerful. We are continuing to refine the UHO formats and are extending the SHOW example to illustrate additional techniques. We propose to use the UHO file format as the standard Gammasphere histogram data format or as a standard export format for users who wish to transport data to formats other than those supported at the Gammasphere facility. We would like to thank the Department of Energy for partial support of this project (DE-FG05-92ER40726). 


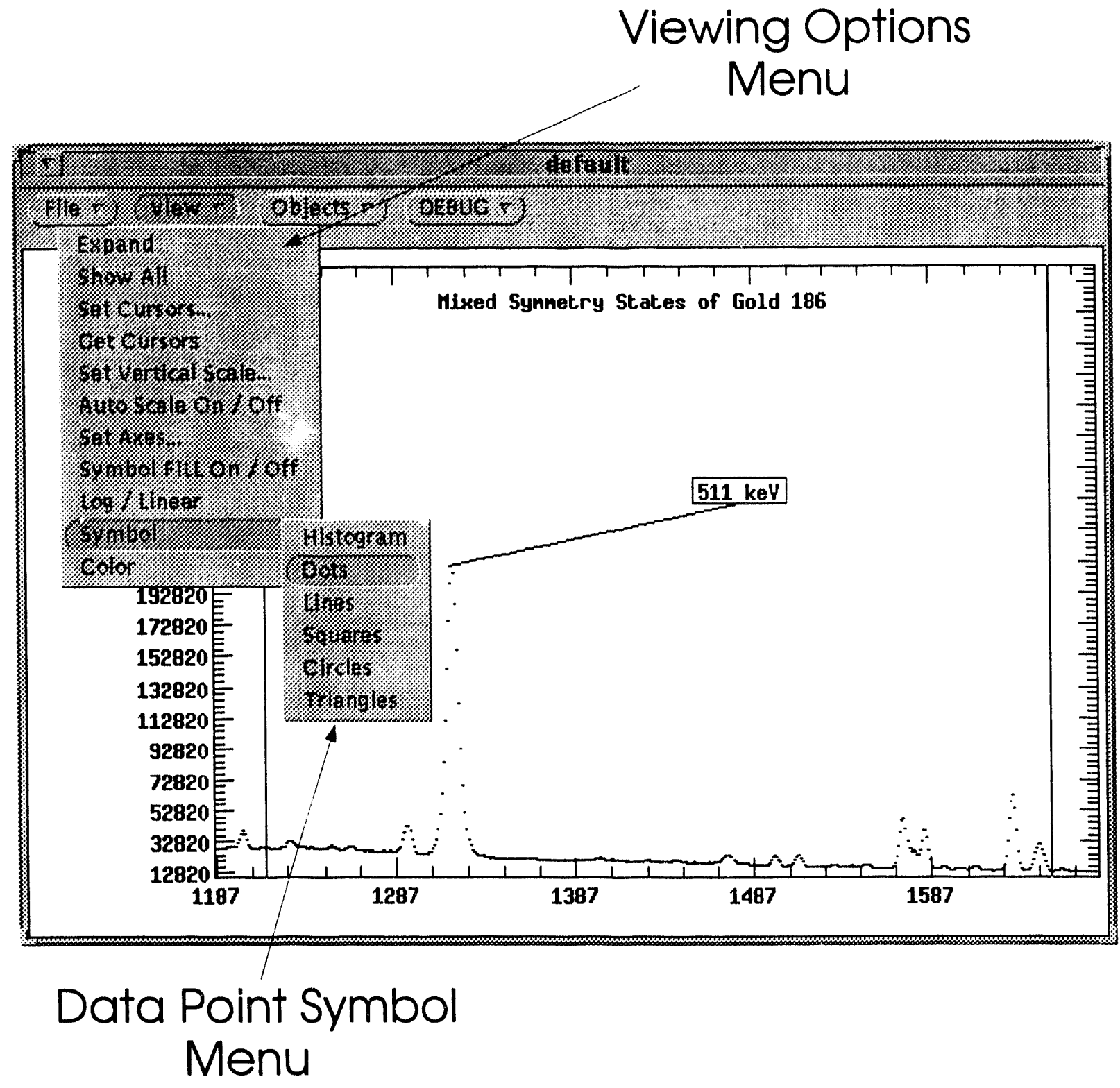




\section{Objects Menu}

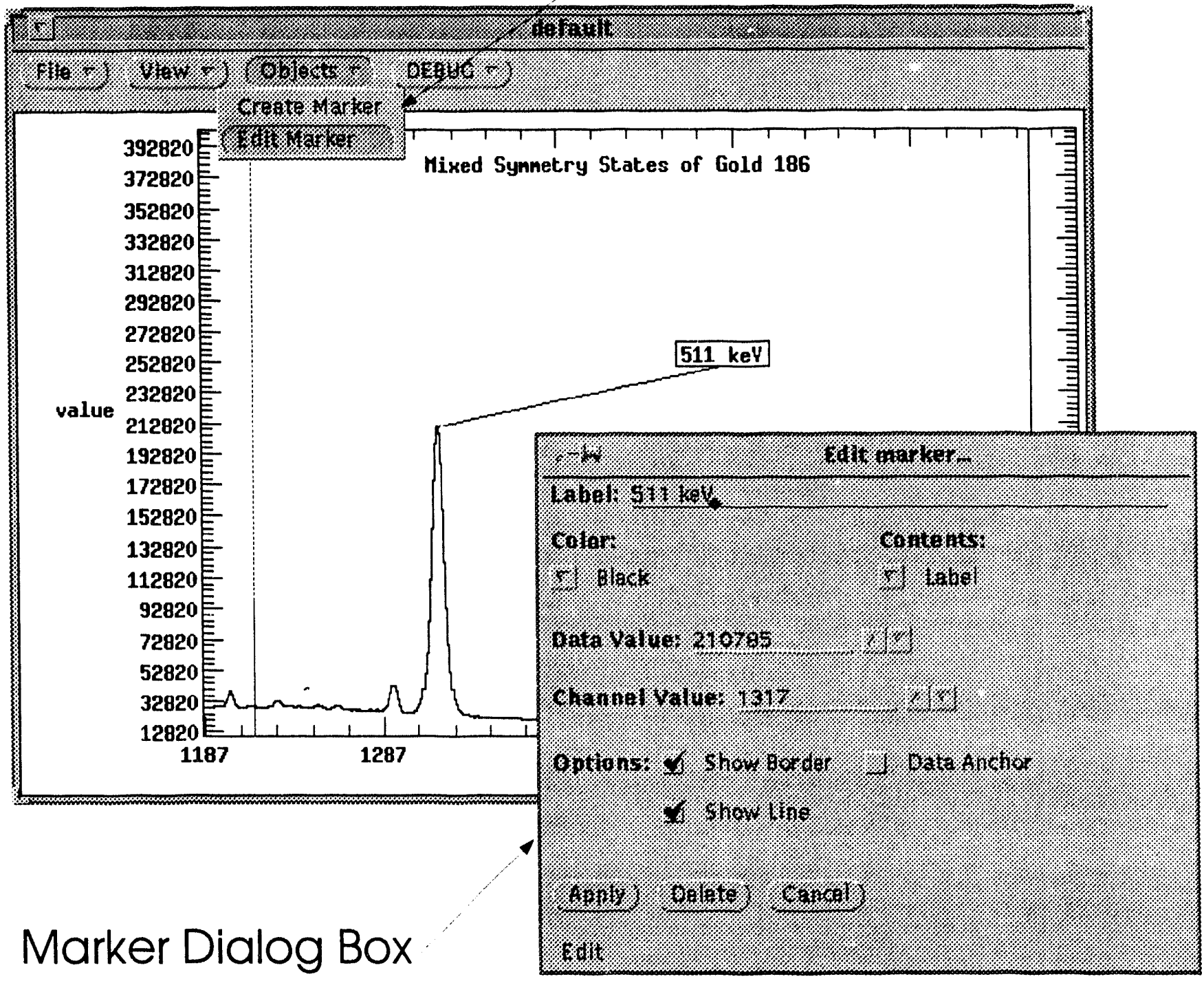




\section{UHO Markers}

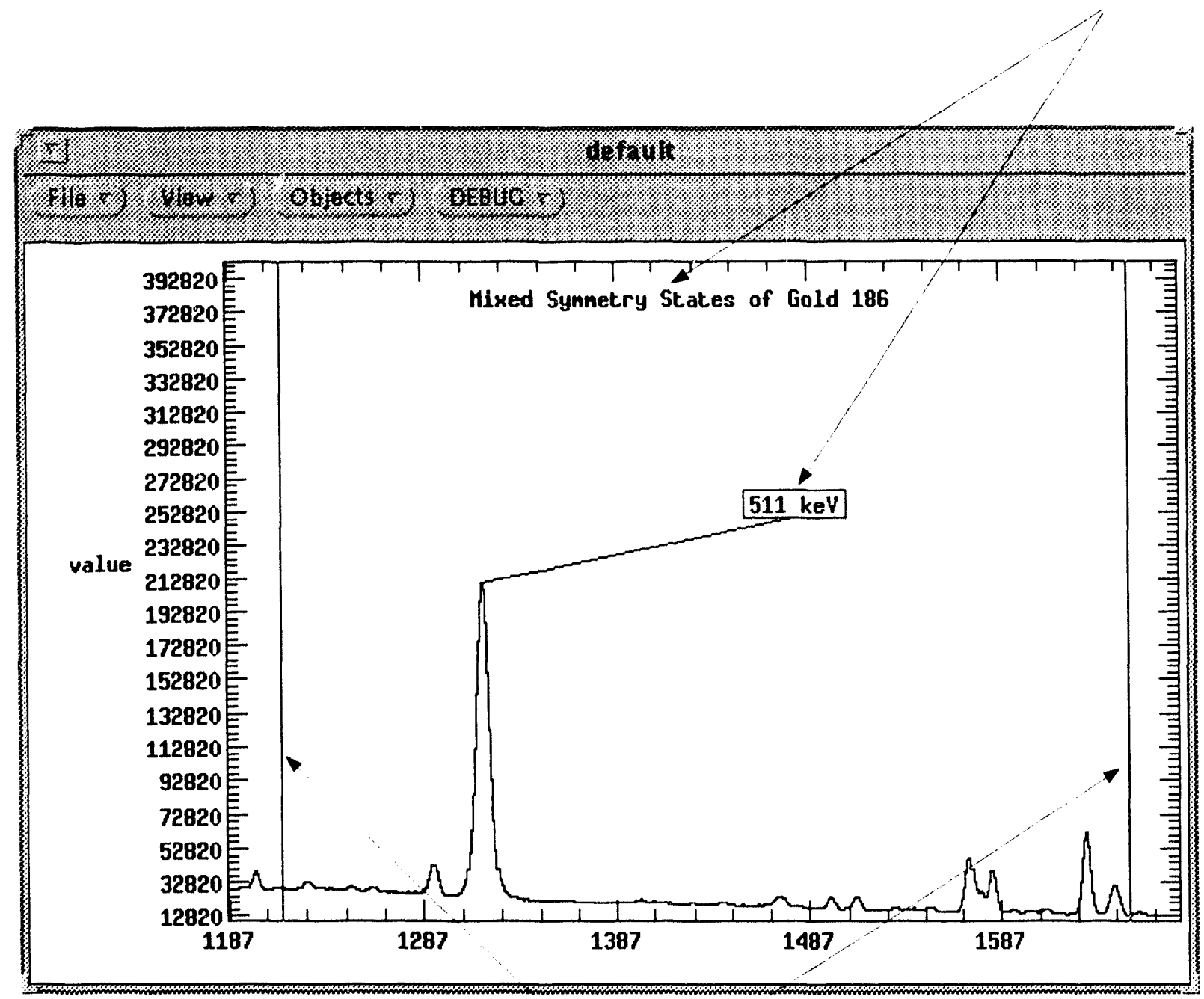

\section{Cursors}




\section{File Manipulation}

Menu

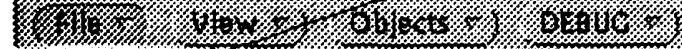

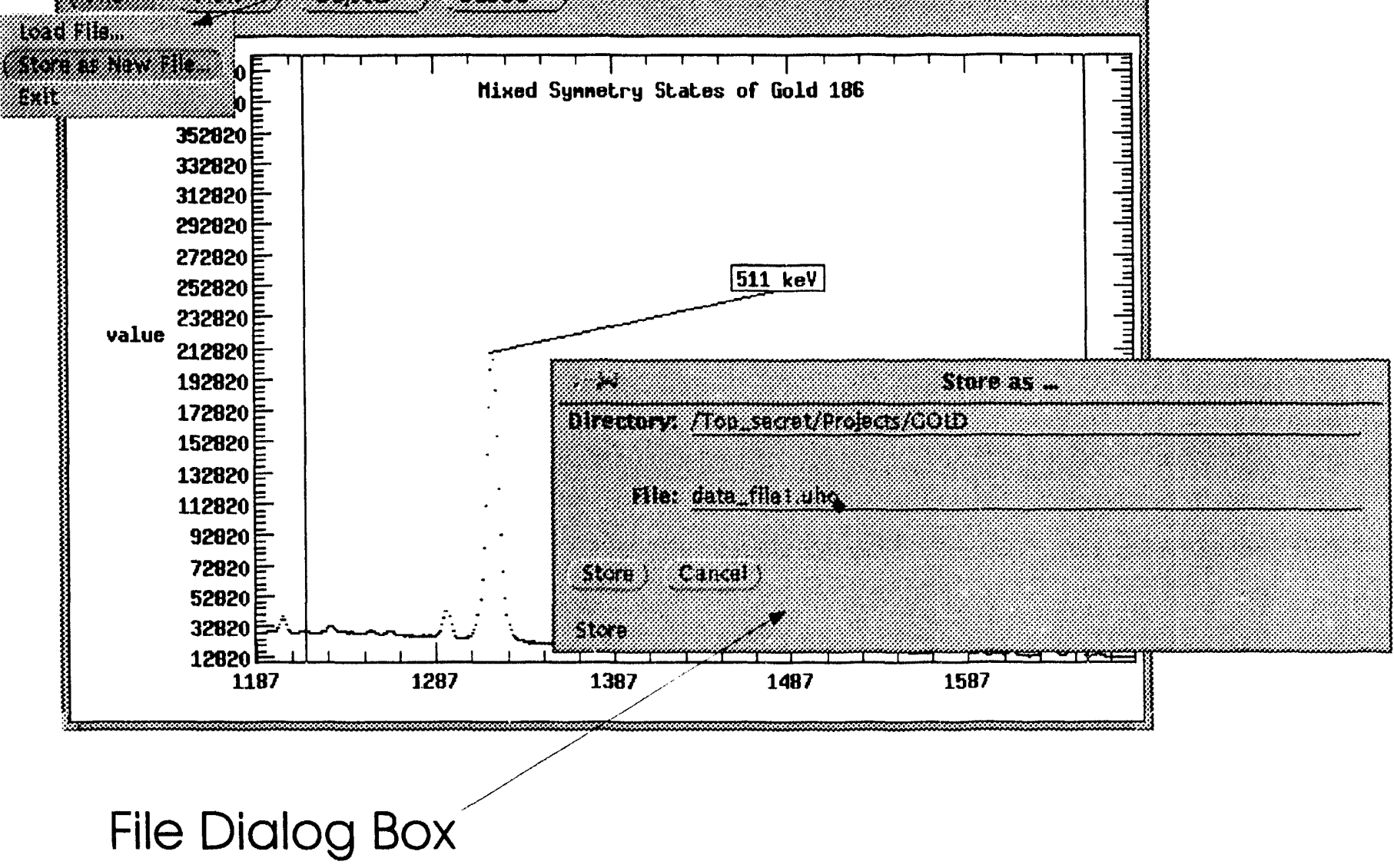




\section{Development of a User Interface Shell}

The complete software environment for the Gammasphere facility consists of a number of major components developed at sites other than the Lawrence Berkeley Laboratory. For example, histogramming software will be contributed by the Oak Ridge National Laboratory, while calibration and simulation software will be developed at Argonne National Lab. Other laboratories and university research groups are also expected to contribute.

The participation of many groups in the development of the Gamasphere software will provide the facility with a wealth of high quality, high performance software tools that would otherwise be unavailable. However, these independently designed components present the user with a significant challenge. Since each has been designed based on a different functional model and since the authors have implemented different user interfaces, the learning curve can be so steep that many of the excellent tools may go essentially unused.

In light of this situation, there is considerable interest in the implementation of a consistent graphical user interface (GUI) for the Gammasphere software environment, particularly for those elements which provide the basic user features such as histogram generation, histogram display and histogram analysis, etc. However, it is unreasonable to expect independent developers to redesign their software to incorporate the same user interface model.

The Oak Ridge National Laboratory histogramming software package (UPAK) is of special interest. In addition to the job of porting the software elements to the SPARC-based machines, it has been pointed out that some effort should be expended in developing a full graphical user interface for UPAK. A subcommittee has been formed to identify the relevant issues and to suggest enhancements where they could significantly improve the usability of the software.

\section{DO_SHELL}

In order to address the concerns of the committee, we are developing an application shell (do_shell) which can be user configured to provide a GUI for any target application operated via a command line interface (e.g., the Oak Ridge Histogramming Package). The do_shell application is an X based Graphical User Interface (GUI) shell program. The current version of do_shell uses the xview toolkit to provide the buttons and other widgets interface to an base application. Interprocess communication is handled with the ToolTalk message services. Do_shell opens a base frame which has a base panel attached to it. The top portion of the base panel has a panel text widget followed by various user widgets. Do_shell currently uses ToolTalk functions to pass 
integer and string arguments to the target application. The ToolTalk routines are user activated by clicking on a button on the do_shell control panel. Below the base panel is a standard tty window where commands may be issued and messages displayed. Do_shell can be configured to automatically launch the target application, effectively hiding the complex relationship between the control shell and the target application. Once configured the target application operates as if it had a GUI rather than a command line interface. In order to support bidirectional comunication with the do_shell interface a target program must support the ToolTalk messageing protocol.

A control flow diagram of do_shell and a target program is shown in figure 1. As discussed, the implementation of the the do_shell application will allow users to operate the UPAK and other software modules without learning the program specific command structure.

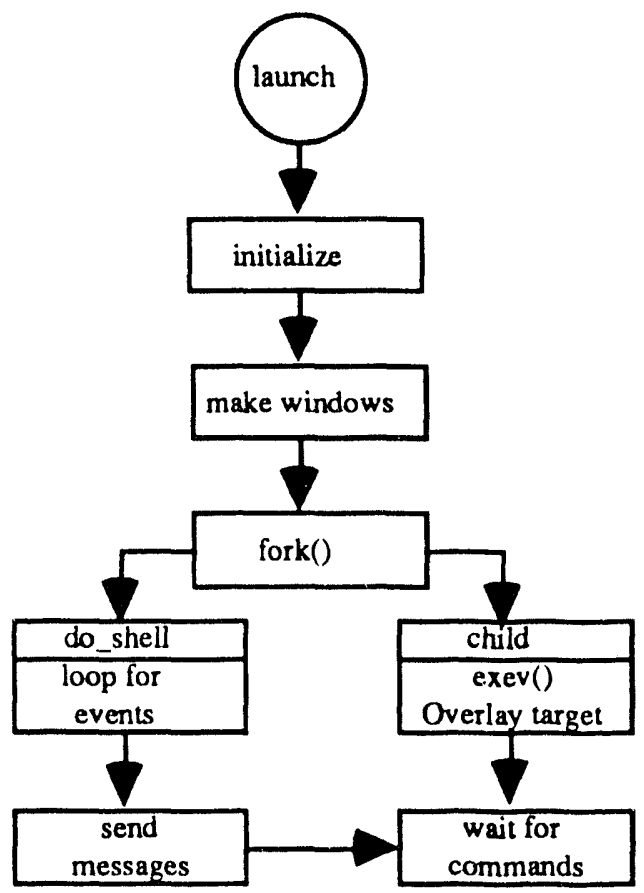

Flow chart for the proposed GUI shell to run on the SPARCstation.

Other areas where the UPAK user interface could be improved include window behavior, printing features and general window manager interoperation. For example, the ability to support simple cut and paste operations from Damm into drawing programs would be a useful extension. 
Such complex enhancements would require some modifications to the UPAK source code and would have to be closely coordinated with the Oak Ridge programming team.

The announcement of the Common Open Software Environment (COSE) in March of 1993 has prompted the Unix user community to rethink its GUI development. It is in this light that our do_shell program is being ported from Xview to the $\mathrm{X}$ toolkit $(\mathrm{Xt})$. Tooltalk will also play a central role in the new COSE consortium and ToolTalk support will be included in MSU application development. 


\section{An FTP Site for Software Development Support}

In order to make the results of our work generally available, we have established a public ftp site at MSU. Final products as well as works-in-progress are posted regularly so that the user community has the most recent versions of the data formats, subroutine libraries and programming examples. In addition, we post information on industry trends and solicit input from the user community. The Site Manual and a current directory for the site are provided in an appendix. 


\section{Testing the ORNL SUNPAK Software}

We have been testing the compatibility of the ported UPAK software package and are providing the test results to ORNL. The most recent test summary is included as an appendix. 


\section{Other Work}

Mixed-symmetry States In Gold 186

Mr. Morris Fields is currently working towards his Masters degree by studying mixedsymmetry states in $\mathrm{Au}^{186}$. He is currently analyzing gamma-ray spectra acquired in an experiment done at the Holifield Heavy Ion Research Facility (HHIRF) at Oak Ridge National Laboratory. Mr. Pete Varmette is analyzing data from a similar experiment to study $\mathrm{Pd}^{106 .}$

Numerical Algorithms for Relativistic Hydrodynamic Collisions

Mr. Hazem Tliemat and Ms. Anita Trevida are collaborating with the MSU Engineering Research Center (ERC) to develop grid-based numerical algorithms to study relativistic hydrodynamic collisions between heavy nuclear. Animated video sequences have been produced and are available from our group or the ERC. 


\section{Publications, Abstracts and Presentations}

\section{Publications}

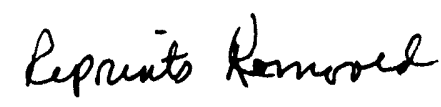

"Performance of a three-element thin film detector," Z. Milosevich, M.L. Muga, R.L. Coldwell, G.J. Bamford, P.S. Haskins and R.B. Piercey, Nucl. Instr. and Meth. in Phys. Res, A320, pp. 471-478. (1992)

"Coulomb Excitation Studies to High Spin In $248 \mathrm{Cm}$," R.B. Piercey, C. Michel, E. Grosse, H. Emling, D. Schwalm, H.J. Wollersheim, J.H. Hamilton, A.V. Ramayya and N. Trautmann, L. Phys. G:Nucl. Part. Phys. 19, (1993), 849-860.

"Multiparameter Data Acquisition System for a Germanium Compton Telescope," J.E. Mckisson, P.S. Haskins, R.B. Piercey, G.W Phillips, and S.E. King, (To be published).

"Design of a Laboratory-Based Germanium Double Compton Telescope," P.S. Haskins, J.E. Mckisson, G.W Phillips, S.E. King, and R.B. Piercey, (To be published).

"Laboratory testing of a Volume-Imaging Germanium Compton Camera," P.S. Haskins, J.E. Mckisson, G.W Phillips, S.E. King, R.A. August, R.B. Piercey, and R.C. Mania (To be published).

\section{Abstracts}

"Gamma-ray Imaging with a Germaniom Double-Compton Telescope," P.S. Haskins, J.E. Mckisson, G.W. Phillips, S.E King, R.B. Piercey, (Presented to the DARPA Nuclear Non-Proliferation Sensors Symposium, Patric AFB, February 10, 1993).

"Covariant, Grid-Based Numerical Algorithms for Nuclear Hydrodynamics," R.B. Piercey, B.K. Soni, H. Tleimat and Anita Trevedi (Presented to the Mississippi State Annual Conference on Differential Equations \& Computational Simulations, Mississippi State University, Mississippi State, MS, March 19, 1993). 
"A Macintosh-Based, Transportable, Multiparameter Data Acquisition System," R.B. Piercey and D.K. Fulton (Presented to IEEE Real Time 93 Conference, Vancouver, CANADA, June, 1993).

"A Universal Histogram Object For Nuclear Science," R.B. Piercey, R.A. Belshe, R.W. MacLeod and R.L. Varner(Presented to IEEE Real Time 93 Conference, Vancouver, CANADA, June, 1993).

"Multiparameter Data Acquisition System for a Germanium Compton Telescope," J.E. Mckisson, P.S. Haskins, R.B. Piercey, G.W Phillips, and S.E. King, (Presented to IEEE Nuclear Science Symposium, San Francisco, CA, Nov., 1993).

"Design of a Laboratory-Based Germanium Double Compton Telescope," P.S. Haskins, J.E. Mckisson, G.W Phillips, S.E. King, and R.B. Piercey, (Presented to IEEE Nuclear Science Symposium, San Francisco, CA, Nov., 1993).

\section{Siminars and Oral Reports}

"Future Computing-OS Wars," Mississippi State University, Mississippi State, MS, April 29.

"Multiparameter Data Acquisition," Jackson State University, Jackson, MS, October 7.

"Report of the Software Working Group Activities to the Gammasphere User's Group," APS Nuclear Physics Division Meeting, Pacific Grove, CA, October 20-23. 


\section{Project Chronology}

March 19, 20 (Mississippi State)

Professor Piercey participated in the rewiew of and attended the "A "'al Conference on Differential Equations \& Computational Simulations" where he presented a payer and chaired a technical session.

\section{March 28-April 1 (Santa Clara, CA)}

Professor Piercey attended the annual SUN Microsystems sponsored developer conference.

April 29 (MSU)

Professor Piercey gave siminar on "Future Computing-OS Wars" for the Physics Department.

May 15 (MSU MS)

Professor Piercey conducted an Oral Exam for Mr Pete Varmette. Mr. Varmette was awarded the Masters Degree for his work in Nuclear Physics.

May 1-15 (MSU MS)

Professor Piercey arranged for Pete Varmette to work with Gerry Fishman and Pat Lestrade at NASA Huntsville in the Summer.

June 6-13 (Vancouver CA)

Professor Piercey attended the IEEE Real Time 93 Conference and presented two Papers.

July 28- August 1 (Berkeley CA)

Professor Piercey attended and chaired the third meeting of the SWG in Berkeley CA.

October 7 (Jackson, MS)

Professor Piercey gave a siminar in the physics department at Jackson State University on Multiparameter Data Acquisition.

October 20-23 (Pacific Grove CA) 
Professor Piercey attended the Division of Nuclear Physics APS meeting and gave a presentation to the GammaSphere Users Group. Also participated in group discussions regarding worksation selection for the the nuclear science community.

November 1-5 (Sanfrancisco CA)

Professor Piercey attended the IEEE Nuclear Science Symposium. Co-prese nted two papers. 


\section{Appendix 1. UHO C Language Header Files}

\section{An example UHO TEXT record}

$\begin{array}{llllllll}\text { TEXT } & 1 & 8192 & & & & & \\ 0 & 0 & 0 & 0 & 0 & 0 & 1 & 0 \\ 0 & 1 & 1 & 0 & 1 & 0 & 0 & 0 \\ 0 & 0 & 1 & 2 & 0 & 0 & 1 & 1 \\ 2 & 1 & 0 & 1 & 1 & 2 & 2 & 3 \\ 0 & 3 & 5 & 4 & 3 & 2 & 2 & 1 \\ 2 & 1 & 5 & 1 & 2 & 6 & 4 & 3 \\ 4 & 3 & 4 & 3 & 1 & 5 & 3 & 6 \\ 1 & 6 & 5 & 7 & 3 & 6 & 4 & 5 \\ 4 & 5 & 5 & 8 & 8 & 4 & 4 & 1 \\ 3 & 4 & 7 & 9 & 4 & 8 & 8 & 7 \\ 11 & 13 & 11 & 8 & 10 & 10 & 7 & 9 \\ 5 & 13 & 11 & 8 & 12 & 9 & 6 & 14 \\ 10 & 9 & 7 & 10 & 10 & 10 & 11 & 14 \\ 3 & 9 & 11 & 13 & 11 & 8 & 12 & 10 \\ 7 & 10 & 12 & 9 & 8 & 14 & 8 & 13 \\ 4 & 9 & 6 & 3 & 4 & 5 & 1 & 5 \\ 1 & 5 & 8 & 7 & 8 & 3 & 3 & 7 \\ 5 & 7 & 6 & 3 & 4 & 5 & 5 & 3 \\ 8 & 4 & 6 & 2 & 2 & 7 & 5 & 5 \\ 7 & 6 & 5 & 3 & 4 & 6 & 7 & 9 \\ 2 & 3 & 5 & 7 & 5 & 2 & 7 & 5 \\ 4 & 6 & 7 & 4 & 7 & 7 & 3 & 5 \\ 4 & 4 & 5 & 6 & 5 & 3 & 3 & 2 \\ 5 & 8 & 3 & 7 & 6 & 3 & 5 & 2\end{array}$

a total of 1024 lines of text 


\section{UHO C Language Programming Structures}

\section{UHO "DATA" record format}

The uho_DATA structure holds the data in memory and is copied to disk when a histogram is saved as a file. The structure is extended to hold the length array as well as the histogram data. The data[1] field contains the first length value (the only one for 1D histogram). For a 1D histogram and a word_size of 4, data[2] would contain the contents of channel 1.

Histogram data records contain the actual histogram data and are used to flag the beginning of a associated records and to specify the nature of the data record. Data records specify the rank or dimension of the histogram file. A rank of one would correspond to single parameter histogram. A rank of two would mean a two parameter histogram and so on. The word size of the histogram is the storage size in bytes for the contents of a particular channel. The format says how the data is arranged in the data record. Following the format field, the length of each parameter is given. When a data record is encountered in a file the following records are interpreted as belonging to that histogram. When a new data record is encountered it marks the beginning of the next histogram. In this way multiple histograms may be included in a single file. Data records specify the rank or dimension of the histogram file.

typedef struct uho_DATA I

$\begin{array}{llll}\text { uhoTag } & \text { rec_tag; } & /^{*} \text { 'DATA' } & * / \\ \text { size_t } & \text { rec_size; } & / * \text { size in bytes of this record } * / & \\ \text { long } & \text { rec_vers; } & / * \text { version of record }(1.0 \mathrm{~B}) & * / \\ \text { long } & \text { rec_ID; } & / * \text { unique ID of record } & * / \\ \text { long } & \text { dt_usr_data; } & / * \text { the usr can store anything here } & * / \\ \text { long } & \text { dt_byte_order; } & / * \text { byte order 0x01020304 } & * / \\ \text { time_t } & \text { dt_creat_time; } / * \text { the creation time } & \\ \text { long } & \text { dt_rank; } & / * \text { identifys the data rank: 1D, 2D,.. } & * / \\ \text { long } & \text { dt_word_size; } / * \text { byte, word, long, etc. }\end{array}$


long

dt_format;

${ }^{*}$ int, float, etc

$*$ /

long

dt_compress;

$I^{*}$ how data is compressed

*1

long

dt_data[1];

/* length array(longs), data follows */

] uho_DATA; 


\section{UHO "TEXT" record format}

typedef struct uho_TEXT \{

$\begin{array}{lll}\text { uho'Tag } & \text { rec_tag; } & \text { * }^{*} \text { TEXT' } \\ \text { char } & \text { data[1]; } & I^{*} \text { rank, len } 1, \text { len2,... lenN, data1, data2... }\end{array}$

] uho_TEXT ;

\section{UHO "ERRS" record format}

typedef struct uho_ERRS ?

\begin{tabular}{|c|c|c|c|}
\hline uhoTag & rec_tag; & \multicolumn{2}{|l|}{ /*'ERRS' } \\
\hline size_t & rec_size; & \multicolumn{2}{|c|}{$I^{*}$ size in bytes of this record } \\
\hline long & rec_vers; & \multicolumn{2}{|c|}{$/ *$ version of structure $(1.0 \mathrm{~B}) * /$} \\
\hline long & rec_ID; & \multicolumn{2}{|c|}{$/ *$ unique ID of record } \\
\hline long & er_usr_data; & \multicolumn{2}{|c|}{ I* The usr can store anything here $^{*}$} \\
\hline long & er_owner_ID; & $I^{*}$ owner ID of record & $* /$ \\
\hline long & er_format; & \multicolumn{2}{|c|}{ /* sqrt, explicit, symmetric, etc } \\
\hline long & er_draw_freq; & to apply during sorting & *I \\
\hline long & er_data[1]; & \multicolumn{2}{|c|}{ /* length array(longs), data follows } \\
\hline
\end{tabular}

\} uho_ERRS ; 


\section{UHO "DSPL" record format}

The uho_DSPL record holds additional drawing control information and the value axes descripters. There should only be one uho_DSPL record even for higher dimensional data.

Histogram "markers" are used to annotate the histogram and can be used to maintain feature lists or simply to add comments to the histogram plots.

typedef struct uho_DSPL 1

\begin{tabular}{|c|c|c|c|}
\hline uhoTag & rec_tag; & /*'DSPL' & *I \\
\hline size_t & rec_size; & $I^{*}$ size in bytes of this record $*$ & */ \\
\hline long & rec_vers; & $/^{*}$ version of structure $(1.0 \mathrm{~B}) *$ & *l \\
\hline long & rec_ID; & $I^{*}$ unique ID of record & *I \\
\hline long & dp_usr_data; & $I^{*}$ the usr can store data here * & *I \\
\hline long & dp_owner_ID; & $/^{*}$ unique $\mathrm{ID}$ of record & *I \\
\hline XRectangle & dp_window_rect; & $/ *$ how to draw the window & *1 \\
\hline XRectangle & dp_display_rect; & $1 *$ where to draw the data & *I \\
\hline struct rgbColor & dp_fore_color; & I* window background color & *I \\
\hline struct rgbColor & dp_back_color; & /* window background color & $* 1$ \\
\hline long & dp_plot_symbol; & /* Dots,Squares,Lines & $* /$ \\
\hline long & dp_symbol_size; & /* symbol size & */ \\
\hline long & dp_symbol_fill; & $I^{*}$ to fill the Plot & *I \\
\hline long & dp_remove_lines; & /* remove hidden lines & *I \\
\hline long & dp_val_min; & $I^{*}$ smallest value displayed & $* 1$ \\
\hline long & dp_val_max; & /* largest value displayed & $* 1$ \\
\hline long & dp_vert_mode; & $/ *$ cLINEAR or cLOG & */ \\
\hline long & dp_view_persp; & $I^{*}$ cHUMAN, cTFLESCOPIC & C..*/ \\
\hline long & dp_view_type; & $I^{*}$ cSURFACE, cSCATTER..* & $*$ I \\
\hline long & dp_maj_intvl; & $I^{*}$ majr intrvl for the val axis & $* /$ \\
\hline long & dp_min_intvl; $/ * \min$ & Ir intrvl for the val axis $*$ / & \\
\hline long & dp_font_name[128]; & $/ *$ specifies font for the axis & $* /$ \\
\hline long & dp_show_grid; & $I^{*}$ says whether to draw grid & $* 1$ \\
\hline long & dp_auto_axes; & $/ *$ automatically adjust axes & $*$ \\
\hline
\end{tabular}




$\begin{array}{llll}\text { long } & \text { dp_auto_scale; } & \text { /* auto adjust vertical scale } & * / \\ \text { long } & \text { dp_draw_axis; } & \text { /* says to draw value axis } & * / \\ \text { long } & \text { dp_axis_label[cMAX_LABEL_LENGTH];/* label } & * /\end{array}$

\} uho_DSPL; 


\section{UHO "PARM" record format}

The uho_PARM record holds the descriptive information for each dimension of the data array. For example, if the rank is 2 , there can be up to 2 uho_PARM records. The uho_PARM record provides a next_PARM field to enable link-list storage in memory.

"Parameter" records are used to specify how to sort event data and interpret and draw the histogram data. There may be no PARM records, or there may be one for each of the parameter or dimensions in the n-dimensional data set (rank of them).

typedef struct uho_PARM \{

\begin{tabular}{|c|c|c|}
\hline uhoTag & rec_tag; & /* 'PARM' \\
\hline size_t & rec_size; & $1^{*}$ size in bytes of this record \\
\hline long & rec_vers; & $I^{*}$ version of structure $(1.0 \mathrm{~B})$ \\
\hline long & rec_ID; & $I^{*}$ unique $\mathrm{ID}$ of record \\
\hline long & pm_usr_data; & $/^{*}$ the usr can store data here \\
\hline long & pm_owner_ID; & $I^{*}$ owner ID of record \\
\hline long & pm_parm_num; & $/^{*}$ belongs to this hist dimension \\
\hline void & $*_{\text {next_PARM; }}$ & $I^{*}$ points to the next PARM record \\
\hline long & pm_sort_parm; & $I^{*}$ which event parm to sort \\
\hline float & pm_sort_gain; & $I^{*}$ gain to apply during sorting \\
\hline long & pm_sort_offset; & ${ }^{*}$ offset to apply during sorting \\
\hline long & pm_next_chan; & 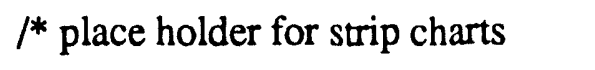 \\
\hline long & pm_disp_min; & $I^{*}$ min channel currently displayed \\
\hline long & pm_disp_max; & /* max channel currently displayed \\
\hline long & pm_curs_min; & $I^{*}$ position of the lower cursor \\
\hline long & pm_curs_max; & $/^{*}$ position of the upper cursor \\
\hline float & pm_axis_rot; & $/ *$ rotation about this axis \\
\hline long & pm_maj_intvl; & $/ *$ major interval for drawing axis \\
\hline long & pm_min_intvl; & $I^{*}$ minor interval for drawing axis \\
\hline char & pm_draw_axis; & $/^{*}$ says to draw the axes \\
\hline
\end{tabular}


char pm_axis_label[cMAX_LABEL_LENGTH]; /* label*/

\} uho_PARM; 


\section{UHO "CALB" record format}

The uho_CALB record holds the optional calibration data for each dimension. For example, if the rank is 2, there can be up to 2 uho_CALB records. The uho_CALB record provides a next_CALB field to enable link-list storage in memory.

typedef struct uho_CALB I

$\begin{array}{llll}\text { uhoTag } & \text { rec_tag; } & / * \text { 'CALB' } & * / \\ \text { size_t } & \text { rec_size; } & / * \text { size in bytes of this record } & * / \\ \text { long } & \text { rec_vers; } & / * \text { version of structure }(1.0 B) * / & \\ \text { long } & \text { rec_ID; } & / * \text { unique ID of record } & * / \\ \text { long } & \text { ca_usr_data; } & / * \text { the usr can store data here } & * / \\ \text { long } & \text { ca_owner_ID; /* unique ID of record } & * / \\ \text { long } & \text { ca_parm_num; } & / * \text { belongs to this hist dimension } & * / \\ \text { void } & * \text { next_CALB; /* points to the next CALB record } * / & \\ \text { long } & \text { ca_use_calb; } & / * \text { says to use the calibration } & * / \\ \text { float } & \text { ca_calb_coef[1]; } & / * \text { calibration coefficient } & * / \\ \text { char } & \text { ca_calb_units[cMAX_LABEL_LENGTH]; } * \text { units } & * / & \end{array}$

] uho_CALB; 


\section{UHO "REGN" record format}

The "regions" structures allows n-dimensional volumes to be specified in the histogram data. This allows ranges and regions of data to be marked along with the specific features identified with the markers. In addition, regions from any open histogram may be specified in the sorting "conditions".

typedef struct uho_REGN \{

$\begin{array}{llll}\text { uhoTag } & \text { rec_tag; } & / * \text { 'REGN' } & * / \\ \text { size_t } & \text { rec_size; } & / * \text { size in bytes of this record } & * / \\ \text { long } & \text { rec_vers; } & / * \text { version of structure }(1.0 \mathrm{~B}) * / & \\ \text { long } & \text { rec_ID; } & / * \text { unique ID of record } & * / \\ \text { long } & \text { rg_usr_data; } & / * \text { the usr can store data here } & * / \\ \text { long } & \text { rg_owner_ID; } / * \text { unique ID of record } & * / \\ \text { void } & \text { *next_REGN; } & / * \text { points to the next REGN record } & * / \\ \text { long } & \text { rg_flags; } & / * \text { visible, selected, etc } & * / \\ \text { long } & \text { rg_rank; } & / * \text { says what kind of region } & * / \\ \text { XColor } & \text { rg_color; } & / * \text { region color } & * / \\ \text { long } & \text { rg_font_num; } & / * \text { font for region text } & * / \\ \text { long } & \text { rg_font_sytle; } & / * \text { font style for region text } & * / \\ \text { long } & \text { rg_font_size; } & / * \text { font size for region text } & * / \\ \text { long } & \text { rg_font_just; } & / * \text { font justification for region text } & * / \\ \text { XRectangle } & \text { rg_rect; } & / * \text { enclosing rect for the region } & * / \\ \text { long } & \text { rg_num_pts } & / * \text { num of points in polygon } & * / \\ \text { XPoint } & \text { rg_point[1] } & / * \text { list of points defines a ploygon } & * / \\ \text { char } & \text { rg_name[cMAX_LABEL_LENGTH]; }{ }^{*} \text { name } & * /\end{array}$

\} uho_REGN; 


\section{UHO "COND" record format}

The uho_COND record holds the description of the condition that is to be applied to a particular ntuple. The fundamental test is whether the ntuple specified by the cond_parm[i] ntuple of rank lies in the region specified by rg_name.

The "conditions" block is a set of conditions or filters which regulate how blocks of events are sorted. These conditions may require that a particular parameter be included in one or more histogram regions, or it may require that a user supplied function evaluate to true.

typedef struct uho_COND \{

\begin{tabular}{|c|c|c|}
\hline uhoTag & rec_tag; & /* 'COND' \\
\hline size_t & rec_size; & $I^{*}$ size in bytes of this record \\
\hline long & rec_vers; & $/ *$ version of structure $(1.0 \mathrm{~B}) * /$ \\
\hline long & rec_ID; & /* unique ID of record \\
\hline long & cd_usr_data; & $I^{*}$ the usr can store data here \\
\hline long & cd_owner_ID; & $I^{*}$ ID of owner record \\
\hline void & *next_COND; & ${ }^{*}$ points to next COND record \\
\hline long & cd_rank; & $1^{*}$ rank of n-tuple test \\
\hline long & cd_mask; & I* bit test mask \\
\hline long & cd_parm_1; & $I^{*}$ parm 1 to test \\
\hline long & cd_parm_2; & $I^{*}$ parm 2 to test \\
\hline long & cd_num_pts; & $I^{*}$ number of points in polygon \\
\hline long & cd_parm[1]; & $/ *$ ntuple for testing \\
\hline char & cd_name[cMA & BEL_LENGTH];;* name of con \\
\hline
\end{tabular}

] uho_COND; 


\section{UHO "MARK" record format}

typedef struct uho_MARK \&

\begin{tabular}{|c|c|c|}
\hline uhoTag & rec_tag; & /*'MARK' $^{*}$ \\
\hline size_t & rec_size; & $I^{*}$ size in bytes of this record \\
\hline long & rec_vers; & $/ *$ version of structure $(1.0 \mathrm{~B}) * /$ \\
\hline long & rec_ID; & $I^{*}$ unique ID of record \\
\hline long & mk_usr_data; & $/ *$ the usr can store data here $* /$ \\
\hline long & mk_owner_ID; & $/^{*}$ unique $\mathrm{ID}$ of record \\
\hline void & *next_MARK; & $I^{*}$ points to the next MARK record \\
\hline long & mk_flags & $/^{*}$ visible, selected, showmode,..... \\
\hline long & mk_anchor, & $/^{*}$ says stick to data or window \\
\hline long & mk_border; & $I^{*}$ specifies border style \\
\hline long & mk_contents; & $I^{*}$ specifies what to show \\
\hline long & mk_x_start; & ${ }^{*}$ chan or $x$ pix location of label \\
\hline long & mk_y_start; & $/^{*}$ val or y pix location of label \\
\hline long & mk_x_stop; & $/^{*}$ chan or $x$ pix location of line \\
\hline long & mk_y_stop; & $/^{*}$ val or y pix location of line \\
\hline XColor & mk_color; & $I^{*}$ marker color \\
\hline long & mk_font_num; & $/^{*}$ foint for marker text \\
\hline long & mk_font_sytle; & $/^{*}$ font style for marker text \\
\hline long & mk_font_size; / & size for marker text \\
\hline & mk_font_just; & $I^{*}$ font justification for marker text \\
\hline char & mk_text[cMAX & EL_LENGTH];/* name \\
\hline
\end{tabular}

\} uho_MARK; 


\section{UHO "ANOT" record format}

typedef struct uho_ANOT ?

$\begin{array}{llll}\text { uhoTag } & \text { rec_tag; } & \text { /* 'ANOT' } & * / \\ \text { size_t } & \text { rec_size; } & \text { /* size in bytes of this record } & * / \\ \text { long } & \text { rec_vers; } & / * \text { version of structure }(1.0 \mathrm{~B}) * / & \\ \text { long } & \text { rec_ID; } & / * \text { unique ID of record } & * / \\ \text { long } & \text { at_usr_data; } & / * \text { the usr can store data here } * / & \\ \text { long } & \text { at_owner_ID; } & / * \text { unique ID of record } & * / \\ \text { void } & \text { *next_ANOT; } / * \text { points to the next ANOT record } * / & \\ \text { char } & \text { at_font_name[128]; } & / * \text { specifies font for the anotation } & * / \\ \text { char } & \text { at_text[1]; } & / * \text { anotation text starts here } & * /\end{array}$

\} uho_ANOT; 


\section{UHO "ADIR" record format}

typedef struct uho_ADIR I

\begin{tabular}{|c|c|c|}
\hline uhoTag & rec_tag; & $I^{*} \mathrm{ADIR}$ \\
\hline size_t & rec_size; & $/^{*}$ size in bytes of this record \\
\hline long & rec_vers; & $f^{*}$ version of structure $(1.0 \mathrm{~B}) * /$ \\
\hline long & rec_ID; & $/^{*}$ unique $\mathrm{ID}$ of record \\
\hline long & ad_usr_data; & $I^{*}$ the usr can store data here $* /$ \\
\hline long & ad_owner_ID; & ${ }^{*}$ ID of parent record \\
\hline void & ${ }^{*}$ next_ADIR; $/^{*}$ & ts to the next ARCHJ record $\quad * /$ \\
\hline long & ad_num_entries; & $/^{*}$ identifys the data rank: $1 \mathrm{D}, 2 \mathrm{D}, .$. \\
\hline long & ad_rank; & $I^{*}$ identifys the data rank: $1 D, 2 D, .$. \\
\hline long & ad_word_size; & $/^{*}$ byte, word, long, etc. \\
\hline long & ad_format; & $/^{*}$ int, float, etc \\
\hline long & ad_compress; & $/^{*}$ how data is compressed \\
\hline ng & ad_data_offset; & $/^{*}$ offset to first data byte \\
\hline no & ad_data_size; & $/^{*}$ size in bytes of data \\
\hline
\end{tabular}

\} uho_ADIR ; 


\section{UHO "ARCH" record format}

typedef struct uho_ARCH (

$\begin{array}{llll}\text { uhoTag } & \text { rec_tag; } & \text { /*ARCH } & * / \\ \text { size_t } & \text { rec_size; } & / * \text { size in bytes of this record } & * / \\ \text { long } & \text { rec_vers; } & / * \text { version of structure }(1.0 \mathrm{~B}) * / & \\ \text { long } & \text { rec_ID; } & / * \text { unique ID of record } & * / \\ \text { long } & \text { ar_usr_data; } & / * \text { the usr can store data here } * / & \\ \text { long } & \text { ar_owner_ID; } & / * \text { ID of parent record } & * / \\ \text { void } & \text { *next_ARCH; } & / * \text { points to the next ARCH record } & * / \\ \text { long } & \text { ar_data[1]; } & / * \text { data follows }\end{array}$

\} uho_ARCH ; 


\section{UHO "FRAM" record format}

typedef struct uho_FRAM l

$\begin{array}{llll}\text { uhoTag } & \text { rec_tag; } & /^{*} \text { 'FRAM' } & * / \\ \text { size_t } & \text { rec_size; } & /^{*} \text { size in bytes of this record } & * / \\ \text { long } & \text { rec_vers; } & /^{*} \text { version of structure }(1.0 \mathrm{~B}) * / & \\ \text { long } & \text { rec_ID; } & /^{*} \text { unique ID of record } & * / \\ \text { long } & \text { fr_usr_data; } & /^{*} \text { the usr can store data here } * / & \\ \text { long } & \text { fr_owner_ID; } & /^{*} \text { unique ID of record } & * / \\ \text { void } & * \text { next_FRAM; } & /^{*} \text { points to the next FRAM record } & * / \\ \text { XRectangle } & \text { fr_rect; } & /^{*} \text { enclosing rect for animation } & * / \\ \text { char } & \text { an_data[1]; } & /^{*} \text { Frame data starts here } & * /\end{array}$

\} uho_FRAM ; 


\section{UHO "ANIM" record format}

typedef struct uho_ANIM !

\begin{tabular}{|c|c|c|}
\hline uhoTag & rec_tag; & ${ }^{*}$ ANIM \\
\hline size_t & rec_size; & $/^{*}$ size in bytes of this record \\
\hline long & rec_vers; & $I^{*}$ version of structure $(1.0 \mathrm{~B}) * /$ \\
\hline long & rec_ID; & $/^{*}$ unique ID of record \\
\hline long & an_usr_data; & /* the usr can store data here $* /$ \\
\hline long & an_owner_ID; & $I^{*}$ unique ID of record \\
\hline void & $*_{\text {next_ANIM; }} /{ }^{*}$ poir & ats to the next ANOT record \\
\hline XRectangle & an_rect; & $/^{*}$ enclosing rect for animation \\
\hline void & *an_frame; & $I^{*}$ pointer to current frame \\
\hline long & an_speed; & $/ *$ frame speed \\
\hline long & an_loop; & $I^{*}$ says whether to loop \\
\hline char & an_font_name[128]; & $/^{*}$ specifies font for the anotation \\
\hline
\end{tabular}

| uho_ANIM ; 


\section{An example UHO histogram hex dump}

$0000000444154410000802 \mathrm{c} 0000000000000000$ /*DATA */

000002000000000000000000000000000000001

00000400000000400000000000000000000 lfff

$0000060001837 b 3000000060000000100000022$

$0000100000000710000010 \mathrm{~b} 0000048 \mathrm{e} 00006074$

$00001200001663 \mathrm{f} 00016 \mathrm{~b} 190001669 \mathrm{f} 0001694 \mathrm{e}$

$00001400001648 f 00016645000165$ ba 00016271

$00001600001609 \mathrm{~d} 00015 \mathrm{e} 8300015 f 1200015 \mathrm{ce} 1$

$0100000000001490000016 \mathrm{f} 0000015300000155$

$01000200000016800000157000001570000015 \mathrm{c}$

$01000400000015900000159000001624453504 \mathrm{c}$ /* DSPL */

$0100060000002 b 0000000000000000000000000$

010010000000000000000000000000000000000

01001200000000000000000000000 ff $00 \mathrm{ff} 00 \mathrm{ff}$

010014000000001000000000000000000000001

$010016000000001001837 \mathrm{~b} 30000000100000001$

$010020000000002000000640000000 \mathrm{a} 00000000$

010022000000000000000000000000000000000

*

$010122000000000000000010000000076616 c 75$ 010124065000000000000000000000000000000 010126000000000000000000000000000000000 *

$01013200000000000000000000000005041524 \mathrm{~d} / *$ PARM */

010134000000090000000000000000000000000

010136000000000000000010000000000000000

$01014003 \mathrm{f} 800000000000000000000000000000$

01014200000 Iffe 000000960000 If 6800000000

$0101440000003 \mathrm{e} 8000000 \mathrm{c} 80000000070617261$ 


\section{Appendix 2. Preliminary SUNPAK Test Results}

\section{DAMM Module Tests}


FOR FILE/DEVICE ASSIGNMENT SYNTAX

\begin{tabular}{|l|l|l|l|}
\hline IN & FIL.EXT & Open N-file (EXT = SPK or HIS) & $y$ \\
\hline OU & FIL.EXT & $\begin{array}{l}\text { Open O-file (EXT = SPK or HIS) - OUTPUT } \\
\text { for SPK only }\end{array}$ & $y$ \\
\hline OU & FIL.SPKNEW & Create and open O-file (SPK-file for output) & $y$ \\
\hline QF & FIL.EXT & $\begin{array}{l}\text { Open Q-file (EXT = SPK or HIS) - } \\
\text { for display only }\end{array}$ & $y$ \\
\hline RF & FIL.EXT & $\begin{array}{l}\text { Open R-file (EXT = SPK or HIS) - } \\
\text { for display only }\end{array}$ & $y$ \\
\hline SF & FIL.EXT & $\begin{array}{l}\text { Open S-file (EXT = SPK or HIS) - } \\
\text { for display only }\end{array}$ & $y$ \\
\hline BAN & FIL & Open FIL.BAN for store recall proj etc & $y$ \\
\hline BAN & FLNEW & Create \& open FIL.BAN for store recall etc & $y$ \\
\hline CLO & F & Closes F-file (where F = N O P Q R S or BAN) & $y$ \\
\hline DFIL & & Displays data files currently open & $y$ \\
\hline CMD & CON & Set CMD-input to be from VDT & $y$ \\
\hline CMD & FIL & Set CMD-input to be from FIL.CMD \& process & y \\
\hline LON/LOF & & Turn output to damm.log ON/OFF (deft= OFF) & $y$ \\
\hline
\end{tabular}

FOR SCREEN SETUP COMMANDS

\begin{tabular}{|l|l|l|l|}
\hline FIGI & & Set screen configuration library to default & $y$ \\
\hline FIGF & FILNAM & Read screen configuration library from ILNAM & y \\
\hline FIG & N & Set current screen configuration to type-N & y \\
\hline DLAF & MS & Set FIG-delay to milliseconds (default=1000) & $y$ \\
\hline DLAD & MS & Set Display-delay to milliseconds (default=500) & $y$ \\
\hline WIN & ID (dflt=1) & Set subsequent displays to be in window-ID & $y$ \\
\hline AXON & ID (dflt) & Enable the drawing of axis for window-ID & $y$ \\
\hline AXOF & ID & Disable the drawing of axis for window-ID & $y$ \\
\hline CMAP & & $\begin{array}{l}\text { Set color map to default } \\
\text { ("takes" after next FIG) }\end{array}$ & y \\
\hline CMAP & FILNAM & $\begin{array}{l}\text { Set color map from FILNAM } \\
\text { ("takes" after next FIG) }\end{array}$ & y \\
\hline REVV & $\begin{array}{l}\text { Reverse all color specs } \\
\text { ("takes" after next FIG) }\end{array}$ & $y$ \\
\hline DLNS & Set \# disp-lines = N - for HELP DDIR \& DSUM & $y$ \\
\hline CURT & LIVE (default) & Set full-wind cursor to track mouse LIVE & y \\
\hline CURT & X & $\begin{array}{l}\text { New full-wind cursor generated via mouse-click } \\
\text { or key }\end{array}$ & $y$ \\
\hline SSI & & Set screen to initial - erase all graphic windows & $y$ \\
\hline
\end{tabular}




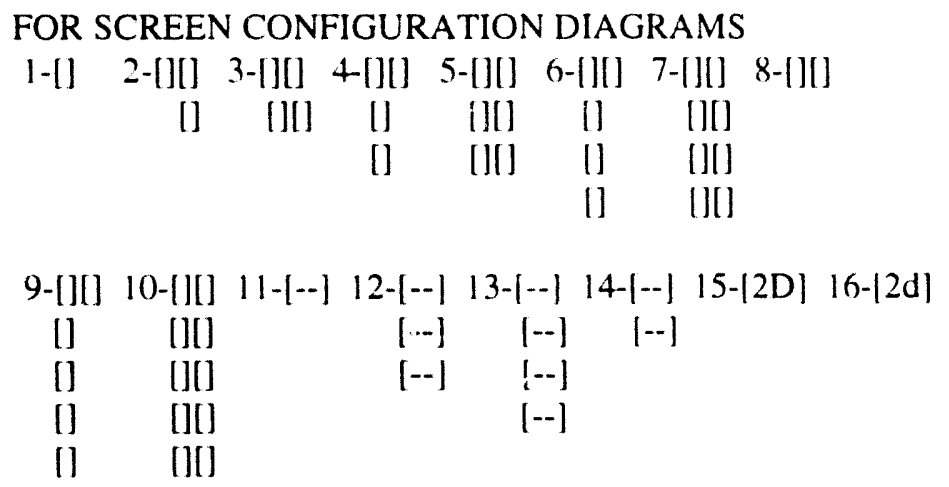

\begin{tabular}{|l|l|l|l|}
\hline DSYM & & Displays list of currently defined sumbols \& values & $y$ \\
\hline LOOP & N & Starts LOOP (executed N-times) N=SYM or CONST & $y$ \\
\hline CMD .... & & Nesting supported & $y$ \\
\hline CMD .... & & \# lines between Ist LOOP \& matching ENDL $=100$ & $\mathrm{y}$ \\
\hline
\end{tabular}


FOR DIRECTORY DISPLAY CMDS.

\begin{tabular}{|l|l|l|l|}
\hline DIR & & Display Input file directory on VDT & y \\
\hline DIR & N & Display Input file directory on VDT & y \\
\hline DIR & O & Display Output file directory on VDT & y \\
\hline DIR & P & Display P-file directory on VDT & y \\
\hline DIR & Q & Display Q-file directory on VDT & y \\
\hline DIR & R & Display R-file directory on VDT & y \\
\hline DIR & S & Display S-file directory on VDT & y \\
\hline DIR & BAN & Display BAN-file directory on VDT & y \\
\hline LDIR ... & & Lists directory on Log-file/device & y \\
\hline DDIR & KF & Display/Log directory of KF-file in more detail & y \\
\hline
\end{tabular}

FOR SOME GENERAL DEFINITIONS

\begin{tabular}{|l|l|}
\hline B1 & Memory Buffer-1 \\
\hline B2 & Memory Buffer-2 \\
\hline ID & The ID-number of histogram to be read \\
\hline NUID & Next ID-number to be assigned to output histogram \\
\hline LO & A tirst-channel-number (usually of a Gate) \\
\hline HI & A last- channel-number (usually of a Gate) \\
\hline FAC & A multiplication factor \\
\hline
\end{tabular}

MEANING OF THE INDIVIDUAL COMMAND-CHARACTERS

\begin{tabular}{|l|l|}
\hline I & Input or read \\
\hline O & Output or write \\
\hline A & Add or accumulate \\
\hline S & Shift (gain shift) \\
\hline GX & Gate on X-parameter (i.e. parameter \# 1) \\
\hline GY & Gate on Y-parameter (i.e. parameter \# 2) \\
\hline I & Buffer-1 \\
\hline 2 & Buffer-2 \\
\hline M & Multiply \\
\hline C & Crunch \\
\hline D & Divide \\
\hline SET & FOR SOME SETUP CMDS \\
\hline
\end{tabular}

COMMANDS FOR SETTING UP NEXT ID\# STD GAIN-SHIFT \& CRUNCH FACTOR .

\begin{tabular}{|l|l|l|l|}
\hline NUID & IV & Set next ID to be used to IV & $y$ \\
\hline IDST & N & Set ID-step to be used in implied I/O loops & $y$ \\
\hline GASP & XIIXI2XFIXF2NCF & Standard gain shift specification & $y$ \\
\hline SIDA & & Says treat 16-bit HIS-file data as signed & y \\
\hline USDA & & Says treat 16-bit HIS-file data as un-signed (deft) & y \\
\hline IO & & FOR I/O SYNTAX & y \\
\hline
\end{tabular}


COMMANDS ' ${ }^{\top} H I C H$ INPUT OR INPUT/OUTPUT I-D HISTOGRAMS

\begin{tabular}{|l|l|l|l|}
\hline I & ID & Input to B1 & $y$ \\
\hline IS & ID & Input to B1 gain shift BI & y \\
\hline IA & IDFAC & Input to B1 B2=B2+FAC*B1 & $y$ \\
\hline ISA & IDFAC & Input to B1 shift BI B2=B2+FAC*BI & y \\
\hline IO & ID & Input to B1 output B1 & y \\
\hline ISO & ID & Input to BI shift BI output B1 & $y$ \\
\hline IO & IDAIDB & Input to B1 output B1 (for ID=IDAIDB) & y \\
\hline ISO & IDAIDB & Input to B I shift output (for ID=IDAIDB) & y \\
\hline O1 & & Output B1 & y \\
\hline O2 & & Output B2 & y \\
\hline
\end{tabular}

FOR 2-D GATING SYNTAX COMMANDS .

\begin{tabular}{|c|c|c|c|}
\hline GY & IDLOHI & Y-gate to B1 & $y$ \\
\hline GYS & IDLOHI & Y-gate to B I shift BI & $y$ \\
\hline GYO & IDL.OHI & Y-gate to BI output B1 & $y$ \\
\hline GYO & IDAIDBLOHI & Y-gate to BI ourput B1 (for ID=IDAIDB) & $y$ \\
\hline GYSO & IDLOHI & Y-gate to B 1 shift \& output B & $y$ \\
\hline GYSO & IDAIDBLOHI & Y-gate to B1 shift \& output B1 (forlD=IDAIDB) & $y$ \\
\hline GYA & IDLOHIFAC & Y-gate to $\mathrm{B} 1 \mathrm{~B} 2=\mathrm{B} 2+\mathrm{FAC} * \mathrm{~B} 1$ & $y$ \\
\hline GYSA & IDLOHIFAC & Y-gate to $\mathrm{B} 1$ shift $\mathrm{B} 1 \mathrm{~B} 2=\mathrm{B} 2+\mathrm{FAC} * \mathrm{~B} 1$ & $y$ \\
\hline $\mathrm{GX}$ & IDLOHI & $\mathrm{X}$-gate to $\mathrm{B} 1$ & $y$ \\
\hline GXS & IDLOHI & $X$-gate to B I shift B1 & $y$ \\
\hline $\mathrm{GXO}$ & IDLOHI & $\mathrm{X}$-gate to B1 output B1 & $y$ \\
\hline $\mathrm{GXO}$ & IDAIDBLOHI & $\mathrm{X}$-gate to B1 output B1 (for ID=IDAIDB) & $y$ \\
\hline GXSO & IDLOHI & X-gate to BI shift \& output B I & y \\
\hline GXSO & IDAIDBLOHI & $\mathrm{X}$-gate to B I shift \& output B I (forlD=IDAIDB) & y \\
\hline GXA & IDLOHIFAC & $\mathrm{X}$-gate to $\mathrm{B} 1 \mathrm{~B} 2=\mathrm{B} 2+\mathrm{FAC}$ * $\mathrm{B} 1$ & y \\
\hline GXSA & IDLOHIFAC & $\mathrm{X}$-gate to $\mathrm{B} 1$ shift $\mathrm{B} 1 \mathrm{~B} 2=\mathrm{B} 2+\mathrm{FAC} * \mathrm{~B} 1$ & $y$ \\
\hline $\mathrm{O} 1$ & & Output B1 & $y$ \\
\hline $\mathrm{O} 2$ & & Output B2 & $y$ \\
\hline
\end{tabular}

FOR PROJECTION SYNTAX COMMANDS

\begin{tabular}{|l|l|l|l|}
\hline PJ & IDBIDDEGR & PROJ TO B1 & $y$ \\
\hline PJS & IDBIDDEGR & Proj to BI shift B1 & $y$ \\
\hline PJO & IDBIDDEGR & Proj to B1 output B1 & $y$ \\
\hline PJO & IDAIDBBIDABIDBDEGR & Proj to B1 output B1 & $y$ \\
\hline PJSO & IDBIDDEGR & Proj to B1 shift \& output B1 & $y$ \\
\hline PJSO & IDAIDBBIDABIDBDEGR & Proj shift output & $y$ \\
\hline PJA & IDBIDDEGRFAC & Proj to BI B2=B2+FAC*B1 & $y$ \\
\hline PJSA & IDBIDDEGRFAC & Proj to B1 shift B1 B2=B2+FAC*B1 & $y$ \\
\hline PJAL & & Project all bananals in currently open & $y$ \\
\hline OI & & Output B1 & $y$ \\
\hline$O 2$ & Output B2 & $y$ \\
\hline
\end{tabular}

(DEGR = Projection-axis angle in degrees)

ID denotes histogram-ID; BID denotes banana-ID 
FOR BUFFER MANIPULATION CMDS

\begin{tabular}{|l|l|l|l|}
\hline M1 & XM & Multiply B1 by XM & $y$ \\
\hline M2 & XM & Multiply B2 by XM & $y$ \\
\hline C1 & ICRUN & $\begin{array}{l}\text { Crunch B1 by ICRUN (standard crunch } \\
\text { unchanged) }\end{array}$ & $y$ \\
\hline C2 & $\begin{array}{l}\text { Crunch B2 by ICRUN (standard crunch } \\
\text { unchanged) }\end{array}$ & $y$ \\
\hline S1 & Shift B1 by standard GASP & $y$ \\
\hline S2 & Shift B2 by standard GASP & $y$ \\
\hline S1 & XI1XI2XF1XF2NC & Shift B1 as specified & $y$ \\
\hline S2 & XI1XI2XF1XF2NCF & Shift B2 as specified & $y$ \\
\hline Z1 & & Zero B1 & $y$ \\
\hline Z2 & & Zero B2 & $y$ \\
\hline A12 & FAC & B2=B2+FAC*B1 & $y$ \\
\hline A21 & FAC & B1=B1+FAC*B2 & $y$ \\
\hline SWAP & & Swap B1 \& B2 & $y$ \\
\hline M2D1 & FAC & B2=(FAC*B2)/B1 & $y$ \\
\hline O1 & & Output B1 & $y$ \\
\hline O2 & & Output B2 & $y$ \\
\hline
\end{tabular}

COMMANDS WHICH LIST DATA ON TERMINAL OR PRINTER.

\begin{tabular}{|l|l|l|l|}
\hline PR1 & & Print B1 & $y$ \\
\hline PR2 & & Print B2 & $y$ \\
\hline D1 & LOHI & Display B1 (channels LO thru HI) & $y$ \\
\hline D2 & LOHI & Display B2 (channels LO thru HI) & $y$ \\
\hline PPL & & $\begin{array}{l}\text { FOR COMMANDS RELATED TO } \\
\text { PRINTER-PLOTS }\end{array}$ & $y$ \\
\hline
\end{tabular}

COMMANDS RELATED TO PRINTER-PLOTS .

\begin{tabular}{|l|l|l|l|}
\hline SKRZ & & Set to skip repeated-zeros for printer plots & y \\
\hline PLRZ & & Set to plot repeated-zeros for printer plots & $y$ \\
\hline PLG & IDLOHINCYC & Input to B1 \& LOG plot & $y$ \\
\hline PLN & IDLOHINCFS & Input to B1 \& LIN plot & $y$ \\
\hline PLG & IDAIDBLOHINCYC & Input to B1 \& LOG plot (for ID=IDAIDB) & y \\
\hline PLN & IDAIDBLOHINCFS & Input to B1 \& LIN plot (for ID=IDAIBD) & $y$ \\
\hline PLG1 & LOHINCYC & Log Printer-plot of B1 & y \\
\hline PLG2 & LOHINCYC & Log Printer-plot of B2 & $y$ \\
\hline PLN1 & LOHINCFS & Linear Printer-plot of B1 & $y$ \\
\hline PLN2 & LOHINCYC & Linear Printer-plot of B2 & $y$ \\
\hline
\end{tabular}

(NCFS $=\#$ of counts full-scale for LIN plots)

$(\mathrm{NCYC}=\#$ of cycles for LOG plots) 
COMMANDS WHICH MODIFY CHANNEL CONTENTS

\begin{tabular}{|l|l|l|l|}
\hline SET1 & ICNYV & Set channel ICN of B1 to YV & $y$ \\
\hline SET2 & ICNYV & Set channel ICN of B2 to YV & $y$ \\
\hline SET1 & LOHIYV & Set channels LO-thru-HI of B1 to YV & $y$ \\
\hline SET2 & LOHIYV & Set channels LO-thru-HI of B2 to YV & $y$ \\
\hline SET1 & LOHIYAYB & Set channels LO-thru-HI of B1 to YA-thru-YB & $y$ \\
\hline SET2 & LOHIYAYB & Set channels LO-thru-HI of B2 to YA-thru-YB & $y$ \\
\hline ADD1 & ICNYV & Add YV to channel ICN of B & $y$ \\
\hline ADD2 & ICNYV & Add YV to channel ICN of B2 & $y$ \\
\hline ADD1 & LOHIYV & Add YV to channels LO-thru-HI of B1 & $y$ \\
\hline ADD2 & LOHIYV & Add YV to channels LO-thru-HI of B2 & $y$ \\
\hline ADD1 & LOHIYAYB & Add YA-thru-YB to channels LO-thru-HI of B1 & $y$ \\
\hline ADD2 & LOHIYAYB & Add YA-thru-YB to channels LO-thru-HI of B2 & $y$ \\
\hline
\end{tabular}

SOME CMDS RELATED TO TESTING

\begin{tabular}{|l|l|l|l|}
\hline GEN & IDKOKXNCH & Generate test spectrum in BI (NCH channels) & $y$ \\
\hline COMP & NCH & Compare first NCH-channels of B1 \& B2 & $y$ \\
\hline SUM1 & LOHI & Display sum of counts LO-thru-HI of B1 & $y$ \\
\hline SUM2 & LOHI & Display sum of counts LO-thru-HI of B2 & $y$ \\
\hline
\end{tabular}

1-D DISPLAY COMMANDS (NORMAL)

\begin{tabular}{|l|l|l|l|}
\hline DLAD & DS & Set Display-delay to MS milliseconds (deft=500) & y \\
\hline LIN/LOG & & Set display to linear/log (default is linear) & y \\
\hline ST /OV & & Set to disp mult hist stacked/overlayed (dflt =OV) & y \\
\hline CAL & ABC & Define energy calibrationE=A+B*Chan+C*Chan**2) & y \\
\hline COL & IJK... & Defines color sequence for display & y \\
\hline KRUD & NC & KRUnch Display(draw I histogram vector per NC chan) & y \\
\hline GWID & WID & Define cursor-mode sum-region width (channels) & y \\
\hline DNOR & LOHI & Normalize displayed data to count-sum of chans LOHI & y \\
\hline DL & LOHI & Set display limits (minmax chan\#) & y \\
\hline DMM & LOHI & Set display limits (minmax counts) & y \\
\hline D & IDLST & Display histogram ID's contained in IDI.ST & y \\
\hline DX & IDLST & Display IDLST (range defined by expand-region) & y \\
\hline SUML & LOHI & Define sum region for SUM command below & y \\
\hline SUM & IDLST & Sum counts (LOHI) (IDLST same as D except C illegal) & y \\
\hline C & & Enter cursor-mode & y \\
\hline SSI & & Set screen to initial - erase all graphic windows & y \\
\hline
\end{tabular}


Cl - FOR 1-D DISPLAY COMMANDS (1-KEY CURSOR)

\begin{tabular}{|c|c|c|}
\hline LF-ARROW & Set expand-region lo-limit & $y$ \\
\hline RT-ARROW & Set expand-region hi-limit & $y$ \\
\hline $\mathrm{E}$ & Expand display & $y$ \\
\hline $\mathrm{V}$ & Make marker display visible/invisible (toggles) & $y$ \\
\hline DN-ARROW & Pan DOWN - move picture so cursor-chan at right-screen & $y$ \\
\hline UP-ARROW & Pan UP - move picture so cursor-chan at left-screen & $y$ \\
\hline $\mathrm{L}$ & Set sum-region lo-limit & $y$ \\
\hline $\mathrm{H}$ & Set sum-region hi-limit & $y$ \\
\hline $\mathrm{G}$ & Set sum-region limits (LO=XCUR HI=XCUR+WID-1) & $\mathrm{y}$ \\
\hline $\mathrm{S}$ & Display sum centroid \& fwhm of sum region & $y$ \\
\hline $\mathrm{A}$ & Display sum centroid \& fwhm of sum region & $y$ \\
\hline $\mathrm{C}$ & Draw peak-marker and display chan\# at cursor pos & $y$ \\
\hline $\mathrm{P}$ & Draw peak-marker and display energy at cursor pos & $y$ \\
\hline 1 & Display/log XCUR YCUR channel\# \& energy & $y$ \\
\hline$Q$ & Quit cursor-mode (return to normal-mode) & $y$ \\
\hline PLON & Turns peak logging (to damm.log) ON & $y$ \\
\hline PLOF & Turns peak logging (to damm.log) OFF (default) & $y$ \\
\hline
\end{tabular}

damm.log may $\because$ read (:kipping prog date \& time) as follows:

READ(LU10)IFLGIDCHENER(FILNAM(I)I=116)

1) (29XA4I102F10.02X16A4)

Where: IFLG = 'MARK' for marked peak log entry

ID $=$ Spectrum ID number

$\mathrm{CH}=$ Peak location in channels

ENER = Peak "energy" from calibration constants

FILNAM $=$ First 64 characters of spk- or his-filename 
2-D DISPLAY COMMANDS (NORMAL)

\begin{tabular}{|c|c|c|c|}
\hline CMAP & & Set color map to default ("takes" after next FIG) & y \\
\hline CMAP & FILNAM & Set color map from FILNAM ("takes" after next FIG) & $y$ \\
\hline ZLEV & $\mathrm{N}$ & Set \# of color/grey-scale intensity levels to $\mathrm{N}$ & y \\
\hline ZLEV & & Set \# of color/grey-scale intensity levels to 10 (dflt) & y \\
\hline GRAS & IJK.. & Set grey-scale values (range ()-10()) \& ZLLEV (\# entries) & $y$ \\
\hline ZINT & COLR & Set 2-D intensity mapping to Color (default) & $y$ \\
\hline ZINT & GREY & Set 2-D intensity mapping to Grey-scale & $y$ \\
\hline ZINT & DOTS & Set 2-D intensity mapping to Dot-matrix $(3 \times 3$ or $5 \times 5)$ & $y$ \\
\hline ZSON & & Z-scale ON - displays counts vs colors legend (default) & $y$ \\
\hline ZSOF & & Z-scale OFF & $y$ \\
\hline ZLOG & & Set 2-D display to log (default) & $y$ \\
\hline $\mathrm{XC}$ & $\mathrm{L}(\mathrm{HHI}$ & Set min \& max X-channel numbers for display & $y$ \\
\hline YC & $\mathrm{LOHI}$ & Set min \& max Y-channel numbers for display & $y$ \\
\hline ZMM & $\mathrm{LOHI}$ & Set min \& max counts/channel for display \& count-sum & $y$ \\
\hline ZMM & L.O & Sets min \& searches for max (semi-autoscale) & $y$ \\
\hline ZMM & & Searches for $\min \& \max \quad$ (full-autoscale) & $y$ \\
\hline $\mathrm{DD}$ & ID & Display 2-D histogram ID from IN-file & $y$ \\
\hline $\mathrm{DD}$ & KFID & Display 2-D histogram ID from KF-file & $\mathrm{y}$ \\
\hline DDX & ID & Display 2-D histogram ID from IN-file (expand region) & $\mathrm{y}$ \\
\hline $\mathrm{DDX}$ & KFID & Display 2-D histogram ID from KF-file (expand region) & $y$ \\
\hline DDID & & Shows ID-number \& Filename for current 2-D display & $y$ \\
\hline ZBL & & Zero in-core BAN-library & $y$ \\
\hline LBL & & List in-core BAN-ID numbers & $y$ \\
\hline $\mathrm{C}$ & & Enter cursor-mode & $\mathrm{y}$ \\
\hline SSI & & et screen to initial - erase all graphic windows & $y$ \\
\hline
\end{tabular}


FOR 2-D DISPLAY COMMANDS ( 1 -KEY CURSOR)

\begin{tabular}{|c|c|c|}
\hline LF-ARROW & Set expand-region lo-left-limit & $y$ \\
\hline RT-ARROW & Set expand-region hi-right-limit & $y$ \\
\hline $\mathrm{V}$ & Make expand markers visible/invisible (toggles) & $y$ \\
\hline 1 & Move display such that cursor is at lo-left & $y$ \\
\hline 2 & Move display such that cursor is at hi-left & $y$ \\
\hline 3 & Move display such that cursor is at hi-right & $y$ \\
\hline 4 & Move display such that cursor is at lo-right & $y$ \\
\hline $\bar{Z}$ & Zero (open) active XY-list & $y$ \\
\hline A & Add point to active $X Y$-list & $y$ \\
\hline $\mathrm{D}$ & Dele nearest point in active $\mathrm{XY}$-list & $y$ \\
\hline $\mathrm{M}$ & Move nearest point in active $X Y$-list to cursor pos & $y$ \\
\hline 1 & Insert a point in active $X Y$-list at cursor position & $y$ \\
\hline $\mathrm{L}$ & List active XY-iist (on VDT) & $y$ \\
\hline $\mathrm{B}$ & Draw active BAN \& BAN's in in-core library & y \\
\hline $\mathrm{G}$ & Prompt for ID \& read into in-core BAN-library & $y$ \\
\hline $\mathrm{O}$ & Open nearest in-core BAN for modificatton & $y$ \\
\hline $\mathrm{S}$ & Prompt for ID \& store in in-core library \& on disk & $y$ \\
\hline $\mathrm{R}$ & Store nearest BAN with original ID & $y$ \\
\hline $\mathrm{F}$ & Remove nearest BAN from in-core library \& erase & $y$ \\
\hline $\mathrm{K}$ & Delete nearest BAN from in-core library and disk & $y$ \\
\hline $\mathrm{T}$ & Totalize counts in nearest BAN (active or not) & $y$ \\
\hline $\mathrm{P}$ & Totalize and \& save X-\& Y-projections on PI.OP.SPK & $y$ \\
\hline 1 & Display XY-coordinates of cursor & $y$ \\
\hline $\mathrm{E}$ & Expand & $y$ \\
\hline Q & Quit cursor-mode (return to normal-mode) & $y$ \\
\hline PLON & Turns position logging (to damm.log) $\mathrm{ON}$ & $y$ \\
\hline PLOF & Turns position logging (to damm.log) OFF (default) & $y$ \\
\hline
\end{tabular}

damm.log may be read (skipping prog date \& time) as follows:

READ(LUIO)IFICIII)XCYC(FHINAM(I)I=116)

10)(29XA4I1()2F10.()2X16A4)

Where: IFLG = 'MAR2' for marked position log entry

ID = Histogram ID number

$X C=X$-position in channels

$\mathrm{YC}=\mathrm{Y}$-position in channels

FILNAM = First 64 characters of spk-or his-filename 
COMMANDS RELATED TO PEAK-FINDING DISPLAY LOGGING

\begin{tabular}{|l|l|l|l|}
\hline FIND & & Turn peak-find \& display ON (BIAS=5.) FWHM=5) & $y$ \\
\hline FIND & BIASFWHM & Turn peak-find \& display ON & y \\
\hline NOFI & & Turn peak-find \& display OFF (default is OFF) & y \\
\hline PLON & & Turns peak logging (to damm.log) ON & y \\
\hline PLOF & & Turns peak logging (to damm.log) OFF (default) & y \\
\hline FPB & & $\begin{array}{l}\text { FOR MANUAL ENTRY OF PEAK BACKGROUND } \\
\text { \& SKIP-REGIONS }\end{array}$ & $y$ \\
\hline PZOT & & Zero the Peak Library & y \\
\hline PK & XWASLOASHI & List of complete peak specifications & y \\
\hline BZOT & & Delete Fixed Background array & y \\
\hline BACK & XIY1 X2Y2.. & XY-points for fixed background & y \\
\hline SKIP & & Without List turns SKIP OFF & y \\
\hline SKIP & $1112 \mathrm{~J} 1 \mathrm{~J} 2 .$. & Up to 4 regions to omit from Fit & y \\
\hline
\end{tabular}

DEFINITION OF FWHM ASYM WLIM ALIM NBC ECAL

\begin{tabular}{|l|l|l|l|}
\hline FW & FNAFWBFWC & Coefficients for standard width function & y \\
\hline ASYM & ASLOASHI & Standard Lo-Side and Hi-Side asymmetries & $y$ \\
\hline WLIM & FWLOFWHI & Variation limit factors for peak widths & $y$ \\
\hline ALIM & FALOFAHI & Variation limit factors for peak asymmetries & $y$ \\
\hline NBC & NBC & Number of power series terms in variable BGD & $y$ \\
\hline WOOD & ON/OFF & Says turn Woods-Saxon background term ON/OFF & $y$ \\
\hline ECAL & ECOECAECB & Coefficients for standard energy calibration & $y$ \\
\hline
\end{tabular}

RELATIVE INTENSITY FIXING COMMANDS

\begin{tabular}{|l|l|l|l|}
\hline RELI & XR & $\begin{array}{l}\text { Specifies that the relative intensity of the library peak } \\
\text { nearest to channel X } 1 \text { s to be R. }\end{array}$ & $y$ \\
\hline RELI & ZOT & Says delete all relative intensity entries & $y$ \\
\hline RELI & OFF & Says turn OFF (disable) relative intensity control & $y$ \\
\hline RELI & ON & Says turn ON (enable) relative intensity control & $y$ \\
\hline
\end{tabular}

VARIATION CONTROL COMMANDS

\begin{tabular}{|c|c|c|c|}
\hline DPX & XSTEPDXMAX & Initial step size and limit for peak pos & $y$ \\
\hline $\mathrm{DEL}$ & DELDELFACNDEL. & Initial step size step size multiplier and & $y$ \\
\hline VB & & Use Variable Background (the default) & $y$ \\
\hline $\mathrm{FB}$ & & Use Fixed Background if available & $y$ \\
\hline VX & KVAR & Kind of variation for peak positions & $y$ \\
\hline VW & KVAR & Kind of variation for peak widths & $y$ \\
\hline VALO & KVAR & Kind of variation for Los-Side asymmetries & $\mathrm{y}$ \\
\hline $\mathrm{VAHI}$ & KVAR & ind of variation for Hi-Side asymmetries & $y$ \\
\hline & $\begin{array}{l}=\text { UIND }- \text { says vary } \mathrm{L} \\
=\text { CIND }- \text { says vary } \\
=\text { ULOC }- \text { says vary } \\
=\text { CLOC }- \text { says vary } \\
=\text { FIX } \quad \text { says keep Fi }\end{array}$ & $\begin{array}{l}\text { nconditionally Independently } \\
\text { onditionally Independently } \\
\text { nconditionally Locked } \\
\text { Conditionally Locked } \\
\text { ed - this the default assignmemt }\end{array}$ & \\
\hline
\end{tabular}


GENERAL. FIT DISPLAY CONTROL COMMANDS

\begin{tabular}{|c|c|c|c|}
\hline WIN & NW & Set subsequent displays to be in window-NW & $y$ \\
\hline LIN/LOG & & Set graphic display to LIN (default) or LOG & $y$ \\
\hline $\mathrm{DMM}$ & MINMAX & $\begin{array}{l}\text { Set display limits (minmax counts) MIN or } \\
\text { MAX }=X \text { says use min or max data value }\end{array}$ & $y$ \\
\hline $\mathrm{DL}$ & ILOIHI & Set display-range (channel\# limits) & $y$ \\
\hline DS & ID & Display spectrum\# ID (range defined by DL) & $y$ \\
\hline DS & IDILOIHI & $\begin{array}{l}\text { Display spectrum\# ID (DL values replaced) } \\
\text { (MAX value of IHI-ILO = 8191) }\end{array}$ & $y$ \\
\hline DSX & ID & Display Data defined by Expand Region & $y$ \\
\hline $\mathrm{C}$ & & Enter 1 -key cursor-mode & $y$ \\
\hline DF & & FOR COMMANDS RELATED TO DISPLAY OF FITS & $y$ \\
\hline WIN & NW & Set subsequent displays to be in window-NW & $y$ \\
\hline MON/MOF & & Turn peak-markers ON/OFF for DF (dflt=ON) & $y$ \\
\hline DFI & & Set to display (DF ) DATAFITBGD (default) & $y$ \\
\hline DPK & & Set to display (DF) DATAFITPEAKSBGD & $y$ \\
\hline DPPB & & Set to display (DF) DATAFIT(PEAKS+BGD)BGD & $y$ \\
\hline DF & & Display Fit (channel-limits given by Fit-range) & $y$ \\
\hline $\mathrm{DF}$ & ILOIHI & Display Fit (channel-limits given by ILOIHI) & $y$ \\
\hline DC & NPK & Display Calculated peak \# NPK+ RESIDUAL & $y$ \\
\hline
\end{tabular}

FIT CONTROL COMMANDS

\begin{tabular}{|l|l|l|l|}
\hline FIT & IDILOIHI & Fit Request - (non-linear gradient search) & y \\
\hline GFIT & IDILOIHI & Fit request - (gaussian method) & y \\
\hline RFIT & IDILOIHI & Resume FIT/GFIT start with Parms from last Fit & y \\
\hline LFIT & IDILOIHI & $\begin{array}{l}\text { Linear Fit - no non-linear search } \\
\text { (Fit-range specified by ILOIHI) }\end{array}$ & y \\
\hline FIT & ID X & Fit Range specified by cursors (Fit Region) & y \\
\hline GFIT & ID X & Fit Range specified by cursors (Fit Region) & y \\
\hline RFIT & ID X & Fit Range specified by cursors (Fit Region) & y \\
\hline LFIT & ID X & Fit Range specified by cursors (Fit Region) & y \\
\hline Cirl/C & & Terminates Fit-in-progress & \\
\hline
\end{tabular}

COMMANDS WHICH DISPI AY PEAKS BGD-POINTS \& FIT-PARMS

\begin{tabular}{|l|l|l|l|}
\hline PRP & XLOXHI & Display peaks from Library in range XLO thru XHI & $y$ \\
\hline PRP & & Display all peaks from Library & $y$ \\
\hline PRB & & Display all fixed background points & $y$ \\
\hline FSTAT & & Display current fit-paramelers & $y$ \\
\hline
\end{tabular}


COMMANDS RELATED TO LIST PRINT OR HARD-COPY

\begin{tabular}{|l|l|l|c|}
\hline KPPL & NONE & Do no printer plots (default) & $y$ \\
\hline & FITS & Plot FITS only & $\mathrm{v}$ \\
\hline & ALL & Plot FITS COMPONENTS and RESIDUALS & $y$ \\
\hline PR & & Print and Plot results of last Fit on printer & $y$ \\
\hline DR & & List results of last Fit on VDT (terminal) & $y$ \\
\hline
\end{tabular}

COMMANDS WHICH SAVE FIT PAREMETERS IN LIBRARY

\begin{tabular}{|l|l|l|l|}
\hline SAV & IJ & Save all Parms from peaks I thru J of last Fit in PK-LIB & $y$ \\
\hline SAX & IJ & Save X-Parms for peaks I thru J of last Fit & $y$ \\
\hline SAW & IJ & Save W-Parms for peaks I thru J of last Fit & $y$ \\
\hline SAL & IJ & Save ASL-Parms for peaks I thru J of last Fit & $y$ \\
\hline SAH & IJ & Save ASH-Parms for peaks I thru J of last Fit & $y$ \\
\hline
\end{tabular}

(If IJ ommitted indicated Parms from ALL peaks are saved)

COMMANDS WHICH SET FIT PARAMETERS

\begin{tabular}{|l|l|l|c|}
\hline SET- & X1X2 & $\begin{array}{l}\text { Set STD WIDTH and ASYM for peaks in range X1-X2 } \\
\text { values (defined by FWA FWB FWC ASLO ASHI) }\end{array}$ & $y$ \\
\hline SETW & X1X2 & Set WIDTH for peaks in range X1-X2 Io STD value & $y$ \\
\hline SETW & X1X2WAWBWC & $\begin{array}{l}\text { Set WIDTH for peaks in range X1-X2 to value defined by } \\
\text { WAWBWC (FWAFWBFWC unchanged) }\end{array}$ & $y$ \\
\hline SETL & X1X2 & Set ASLO for peaks in range X1-X2 to STD value & $y$ \\
\hline SETL & X1X2ASLOT & Set ASLO=ASLOT for peaks in range X1-X2 & $y$ \\
\hline SETH & X1X2 & Set ASHI for peaks in range X1-X2 to STD value & $y$ \\
\hline SETH & X1X2ASHIT & Set ASHI=ASHIT for peaks in range X1-X2 & $y$ \\
\hline
\end{tabular}

(If $\mathrm{X} 1 \mathrm{X} 2 .$. omitted indicated Parms for ALL peaks are set)

$$
\begin{aligned}
& \text { LIST OF DEFAULT PARAMETER VALUES } \\
& \mathrm{DEL}=0.05 \quad \text { FWLO }=0.5 \quad \mathrm{VX}=\mathrm{CIND} \quad \mathrm{NBC}=2 \\
& \text { DELFAC }=0.25 \quad F W H I=2.0 \quad \mathrm{VW}=\mathrm{CLOC} \quad W O O D=0 \mathrm{FF} \\
& \text { NDEL }=1 \quad \text { FALO }=0.5 \quad \text { VALO }=\text { FIX } \quad \mathrm{KPPI} .=\text { NONE } \\
& X S T E P=(0.5 \quad F A H I=2 .() \quad V A H I=F I X \\
& \text { DXMAX }=5.0 \quad \text { ASLO }=0.0 \\
& \mathrm{ASHI}=() .()
\end{aligned}
$$


FOR I-KEY (OR 2-KEY) CURSOR REIATED (OMMANI)S

Type $C$ in enter cursor-mode

\begin{tabular}{|c|c|c|}
\hline P/UP & Add/Delete peak to Library (pos specified hy cursor) & $y$ \\
\hline $\mathrm{M}$ & Move nearest displayed peak to cusor pos (FW ASYM unchanged) & $y$ \\
\hline $\mathrm{X} / \mathrm{UX}$ & Fix/Free peak position (for displayed peak nearest to cursor) & y \\
\hline W/UW & Fix/Free peak width (for displayed peak nearest to cursor) & $y$ \\
\hline L/UL. & Fix/Free L.o-Side ASYM (for displayed peak nearest lo cursor) & $y$ \\
\hline $\mathrm{H} / \mathrm{UH}$ & Fix/Free Hi-Side ASYM (for displayed peak nearest to cursor) & y \\
\hline $\mathrm{O} / \mathrm{UO}$ & Turn peak ON/OFF (for displayed peak nearest to cursor) & y \\
\hline $\mathrm{B} / \mathrm{UB}$ & Add/Delete background point at cursor position & y \\
\hline LF-ARO & Set Expand Region Lo-Limit & y \\
\hline RT-ARO & Set Expand Region Hi-Limit & y \\
\hline 1 & Set Fit Region Lo-Limit & y \\
\hline 1 & Set Fit Region Hi-Limit & y \\
\hline 1 & Display chan\# cursor $Y$-value chan contents & y \\
\hline $\mathrm{S}$ & Disp sum cent \& f whm of Fit-Reg - DAT(|,1)AT(|) defines B(GD) & $y$ \\
\hline A & Disp sum cent \& fwhm of Fit-Reg - CUR(|)CUR(l) defines BGD & y \\
\hline$Q$ & Return from cursor control routine & $y$ \\
\hline $\mathrm{E}$ & Expand display (region defined by $<-->$ ) & y \\
\hline
\end{tabular}

HISTOGRAM XFER COMMANISS

\begin{tabular}{|c|c|c|c|}
\hline $\mathrm{HOU}$ & FII NAM & Open FILNAM for histogram ()utpul & y \\
\hline GSX & $\times 11 \times 12 \times 1.1 \times 12$ & Define $X$-gain-shift & $y$ \\
\hline GSY & YIIYIL YFIYF2 & Define Y-gain-shift & $y$ \\
\hline GSXOF/GSYOF & & Turn OFF X/Y-gain-hift & $y$ \\
\hline SNEG OFF & & Turn OFF reset of neg lobit out data (default) & $y$ \\
\hline SNEG IV & & $\begin{array}{l}\text { Says set negative 16-bit output data to IV (you } \\
\text { MUST use SIDA mode for this to work!!) }\end{array}$ & $y$ \\
\hline HSTA & & Shows tiles open \& gain-shift data & $y$ \\
\hline $\mathrm{HCOP}$ & IDIII)( $<<\mathrm{HI}>$ & Copy F*IDI (Input) In II)() (O)utpul) & $y$ \\
\hline $\mathrm{HADD}$ & II)III $)(\mathrm{HI}>\mathrm{F}(\mathrm{O})$ & Add $F I * I D I\left(0 F()^{*} I D()\right.$ & $y$ \\
\hline HDIV & II)III)( $)-1 \mathrm{I}$ & Div lit*II)l hy IDO) de sale in II)( ) & $y$ \\
\hline SHIF & II)III)( ) & Gain-shift IDI \& yfure in II)() & $\mathrm{y}$ \\
\hline SHAD & IDIII ()$<\mathrm{FI}><\mathrm{H}()>$ & Gain-shift IDI \& add (I) II)( ) & $y$ \\
\hline HSET & IID()IV & Set IDO on (output file to IV & $\mathrm{y}$ \\
\hline HZOT & II)( & Set IDO on Output file to () & $y$ \\
\hline
\end{tabular}

SPK-SUMS HIS-SUMS ANI) I)IRL CTORY (OMMANI)S

\begin{tabular}{|c|c|c|c|}
\hline DIJIR & $\mathrm{Kl}$ &  & $y$ \\
\hline DSLM & $\mathrm{KF}$ & $\begin{array}{l}\text { Displays count-sum of all histograms in } \\
\text { KF-file Where KF has same meaning as above }\end{array}$ & $y$ \\
\hline
\end{tabular}


MOUSE BUTTON DEFINITIONS (when in I-KEY cursor mode)

\begin{tabular}{|c|c|c|c|}
\hline BUD & LMR & Defines LeftMiddleRight buttons for cursor in D-window & $\mathrm{y}$ \\
\hline BUDD & LMR & Defines LeftMiddleRight buttons for cursor in DD-window & $y$ \\
\hline BUDS & LMR & defines LeftMiddleRight buttons for cursor in DS-window & $y$ \\
\hline BUD & LHS & $\begin{array}{l}\text { Example (cursor in 1-D) L-butt sets sum-reg lo-limit M-butt } \\
\text { sets sum-reg hi-limit R-butt requests S-sum }\end{array}$ & $\mathrm{y}$ \\
\hline BUDD & ATZ & $\begin{array}{l}\text { Example (cursor in 2-D) L-butt adds banana points M-butt } \\
\text { totalizes enclosed counts R-butt zots banana }\end{array}$ & $y$ \\
\hline $\mathrm{XY}$ & & FOR XY-LINE DISPLAY COMMANDS & $y$ \\
\hline $\mathrm{XYF}$ & filename & Opens $X Y$-file and reads in all data & $y$ \\
\hline $\mathrm{XYI}$ & & Displays XY IDs which have been read in & $y$ \\
\hline XYD & I J K L & $\begin{array}{l}\text { Displays XY data for IDs (I J K L ..) into active window } \\
\text { (default = window I or set by WIN command) }\end{array}$ & $\mathrm{y}$ \\
\hline$X Y P$ & I J K L... & $\begin{array}{l}\text { Same as XYD except that XY-points are shown in addition to } \\
\text { the connecting vectors XYD or XYP with no ID-list uses } \\
\text { previous ID-list }\end{array}$ & y \\
\hline $\mathrm{XYC}$ & $\mathrm{KOL}$ & 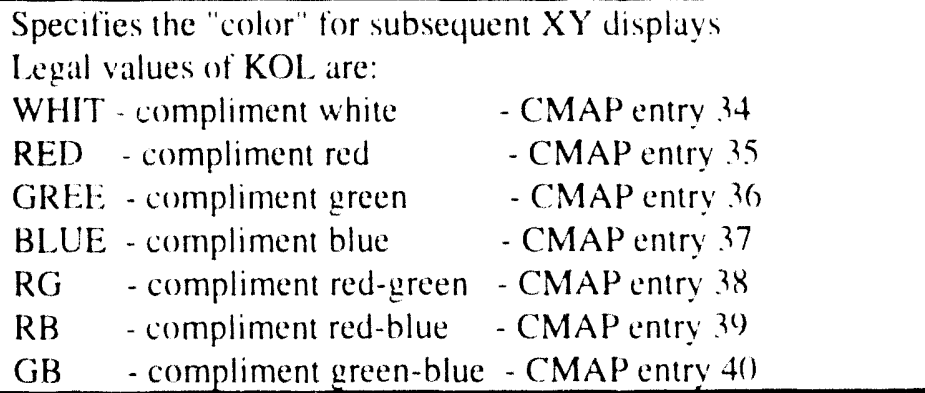 & y \\
\hline
\end{tabular}

Note: Displays are done in "compliment mode" on that displaying the samey

ID a second time will erase it displaying a third time will show it etc. 
LABELING COMMANDS

\begin{tabular}{|c|c|c|c|}
\hline LA7 & text & Adds line of "text" to LA7 label & $y$ \\
\hline LAB & text & Adds line of "text" to LA8 label & y \\
\hline LA9 & text & Adds line of "text" to LA9 label & $y$ \\
\hline LAZ7 & & Deletes all lines of I.A7 label & $y$ \\
\hline LAZ8 & & Deletes all lines of LA8 label & $y$ \\
\hline LAZ9 & & Deleted all lines of LA9 label & $y$ \\
\hline LAL. & & Displays current labels - LA7 LA8 LA9 & $\mathrm{y}$ \\
\hline LAC & $\mathrm{KOL}$ & $\begin{array}{ll}\text { Specifies label color for subsequent displays } \\
\text { Legal values of KOL are: }\end{array}$ & \\
\hline
\end{tabular}

Label Display Commands (1-key cursor-mode)

\section{KEY}

\begin{tabular}{|c|c|c|}
\hline 7 & Displays LA7 at cursor location (compliment mode) & $y$ \\
\hline 8 & Displays LA8 at cursor location (compliment mode) & y \\
\hline 9 & Displays LA9 at cursor Iocation (compliment mode) & y \\
\hline () & Locks previously displayed labels against subsequent change & y \\
\hline
\end{tabular}

(i.e. all labels displayed in a given window will be fixed).

(enables the drawing of additional labels).

Label Pointer Commands (1---key cursor-mode)

$\mathrm{KEY}$ ACTION

\begin{tabular}{|l|l|l|}
\hline$=$ & Adds a vector point to current list and draws point or line & $y$ \\
\hline- & Deletes last vector point in current list and erases line & $y$ \\
\hline$;$ & Locks current vector list against subsequent change and & $y$ \\
\hline & enables new list & $y$ \\
\hline
\end{tabular}

$y$ : The command is working.

The tables were erenerated according 101993 releatse of Damm. 


\section{LEMO Module Tests}

2. LEMO Module Tests

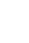

$$
-
$$

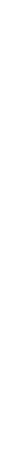


CODE CONTENTS

\begin{tabular}{|l|l|l|}
\hline ASN & File and Tape opening \& closing commands \\
\hline TAPE & & Tape control commands \\
\hline COPY & Copy and Modify-Copy commands \\
\hline CMD & & Command-file operations \\
\hline EVNT & & Event-file Exam \& Copy Commands \\
\hline MISC & & Miscellaneous \& non-standard tape processing commands \\
\hline DISP & commands which display Data-records \\
\hline
\end{tabular}

COMMANDS FOR OPENING \& CLOSING FILES \& TAPES .

\begin{tabular}{|l|l|l|l|}
\hline IN & rstx & Specifies Tape (rst0 rst etc.) for INPUT & $Y$ \\
\hline OUX & rsty & Specifies Tape (rst0 rst etc.) for OUTPUT-X $(X=13)$ & $?$ \\
\hline CLI & & Close INPUT & $?$ \\
\hline CLOX & & Close OUTPUT-X $(X=13)$ & $Y$ \\
\hline ULI & & Unload and close INPUT & $Y$ \\
\hline ULOX & & Unload and close OUTPUT-X (X=1 3) & $?$ \\
\hline MILF & FILE.MIL & Read \& process FILE.MIL (required for Modify-Copy) & $?$ \\
\hline CMDF & FILE.CMD & Assign FILE.CMD as CMD-file (not read yet) & $Y$ \\
\hline END & & Terminates Program & $Y$ \\
\hline
\end{tabular}

TAPE CONTROL COMMANDS

\begin{tabular}{|l|l|l|l|}
\hline RDI & $\mathrm{N}$ & Read N records from INPUT & $\mathrm{Y}$ \\
\hline RDOX & $\mathrm{N}$ & Read N records from OUTPUT-X (X=1 3) & $?$ \\
\hline FRI & $\mathrm{N}$ & Forward N records on INPUT & $\mathrm{Y}$ \\
\hline FROX & $\mathrm{N}$ & Forward N records on OUTPUT-X $(\mathrm{X}=1$ 3) & $?$ \\
\hline BRI & $\mathrm{N}$ & Backup N records on INPUT & $\mathrm{Y}$ \\
\hline BROX & $\mathrm{N}$ & Backup N records on OUTPUT-X $(\mathrm{X}=1$ 3) & $?$ \\
\hline FFI & $\mathrm{N}$ & Forward N files on INPUT & $\mathrm{Y}$ \\
\hline FFOX & $\mathrm{N}$ & Forward N files on OUTPUT-X (X=1 3) & $?$ \\
\hline BFI & $\mathrm{N}$ & Backup N files on INPUT & $\mathrm{Y}$ \\
\hline BFOX & $\mathrm{N}$ & Backup N files on OUTPUT-X (X=1 3) & $?$ \\
\hline RWI & & Rewind INPUT & $\mathrm{Y}$ \\
\hline RWOX & & Rewind OUTPUT-X (X=1 3) & $?$ \\
\hline BTI & & Go to bottom of INPUT (to DBL-EOF backup I EOF) & $\mathrm{Y}$ \\
\hline BTOX & & Go to bottom of OUTPUT (to DBL-EOF backup I EOF) & $?$ \\
\hline CLI & & Close INPUT & $\mathrm{Y}$ \\
\hline CLOX & & Close & $?$ \\
\hline ULI & & Unload and close INPUT & $\mathrm{Y}$ \\
\hline ULOX & & Uuload and close OUTPUT-X (X=1 3) & $?$ \\
\hline FIND & $\mathrm{N}$ & Find HEADER-\# N (does I BACKSPACE if found & $\mathrm{Y}$ \\
\hline DTIT & & Displays next TITLE \& HEADER-\# \& backspaces & $\mathrm{Y}$ \\
\hline
\end{tabular}


COMMANDS FOR COPY \& MODIFY-COPY

\begin{tabular}{|l|l|l|l|}
\hline COPY & N & Copy N files from INPUT to OUTPUT-1 & $?$ \\
\hline CREC & N & Copy N records from INPUT to OUTPUT-1 & $?$ \\
\hline CC & & Continue COPY - saves file- or record-count & $?$ \\
\hline EOFX & & Write EOF on OUTPUT-X (not normally needed) & $?$ \\
\hline MILF & FIL & Read FIL.MIL file (required for MOC) & $?$ \\
\hline UCOM & TEXT & Send TEXT to "USERCMP" & Y \\
\hline INIT & & Resets Modify-Copy Input \& Output buffers & Y \\
\hline RECO & LBYT & Output-RECL (BYTES) (DFLT=8192)(MOC mode only) & $?$ \\
\hline ZBUC & & Zero total Input \& Output buffer counters & Y \\
\hline MOC & N M & Modify-Copy (N-files/M-records - Ist to occur) & $?$ \\
\hline MOCO & NM & Modify-Copy-Continue (waits for tape) & $?$ \\
\hline MOCE & N M & Modify-Copy (END on request complete) & $?$ \\
\hline Ctrl/C & & To interrupt READ or COPY process & Y \\
\hline END & & Terminates Program & Y \\
\hline
\end{tabular}

COMMANDS RELATED TO COMMAND-FILES

\begin{tabular}{|l|l|l|l|}
\hline CMD & FILE & - Assign FILE.CMD as CMD-file (not read yet) & Y \\
\hline CCMD & & - Continue reading CMDS from CMD-file & Y \\
\hline CLCM & & - Continue with last CMD from file (backspaces) & Y \\
\hline CCON & & - Continue reading CMDS from CON: & $Y$ \\
\hline MSG & TEXT & - Display TEXT (44 bytes) on CON: & $Y$ \\
\hline END & & - Terminates program & $Y$ \\
\hline
\end{tabular}

COMMANDS FOR EXAMINATION AND COPY-TO-TAPE OF EVENT-LIST FILES.

\begin{tabular}{|l|l|c|l|}
\hline INFI & filname & - Specify input file for exam (RDI) \& copy-io-tape & Y \\
\hline HTIT & TITLE & - TITLE contains title for next tape header & Y \\
\hline HNUM & HN & - HN specifies next tape header number to use & Y \\
\hline HOUT & & - Outputs tape header and increments HN & Y \\
\hline
\end{tabular}


MISCELLANEOUS \& NON-STANDARD TAPE RELATED COMMANDS

\begin{tabular}{|l|l|l|l|}
\hline LO01 & SKIP NPPE & Specify non-standard Input Tape (see HANDBOOK) & $?$ \\
\hline L002 & & Specify HHIRF standard input tape (default) & $?$ \\
\hline SWAB & & Says byte-swap input records (see document) & $?$ \\
\hline SWOF & & Says byte-swap off (default) & Y \\
\hline SHON & & Says byte-swap headers once more than data & Y \\
\hline SHOF & & Says byte-swap headers \& data the same way & Y \\
\hline RBON & & Turn calls to REBUF ON & Y \\
\hline RBOF & & Turn calls to REBUF OFF (default) & Y \\
\hline UPOF & & Turn User-processing OFF (default) & Y \\
\hline UPON & & Turn User-processing ON (NPRAW=() RECL=8192 bytes) & Y \\
\hline UPON & NPARU & Turn User-processing ON ( RECL=8192 bytes) & Y \\
\hline UPON & NPRAW RECL & Turn User-processing ON & ON \\
\hline RECO & NBYTES & Specify output record length (dflt=8192 bytes) & Y \\
\hline FCOP & FILNAM.EXT & Copy FILNAM.EXT to OUTPUT-1 (80 byte ASCII) & $?$ \\
\hline TCOP & FILNAM.EXT & Copy INPUT-TAPE to FILNAM.EXT (80 byte ASCII) & $?$ \\
\hline STEX & FILNAM.EXT & Store TEXT-BLKS on FlLNAM.EXT & $?$ \\
\hline END & & Terminates Program & Y \\
\hline
\end{tabular}

COMMANDS FOR READING \& DISPLAY OF DATA-RECORDS

\begin{tabular}{|l|l|l|l|}
\hline RDI & N & Read N records from INPUT & Y \\
\hline RDOX & N & Read N records from OUTPUT-X $(X=13)$ & $?$ \\
\hline PEV & IA IB & Print 16-bit word IA thru IB in EVENT format & $?$ \\
\hline DEV & IA IB & Display 16-bit word IA thru IB in EVENT format & $?$ \\
\hline PZ & IA IB & Print 16-bit word IA thru IB in HEX format & Y \\
\hline DZ & IA IB & Display 16-bit word IA thru IB in HEX format & Y \\
\hline PA & IA IB & Print I6-bit word IA thru IB in ASCII format & Y \\
\hline DA & IA IB & Display 16-bit word IA thru IB in ASCII format & Y \\
\hline PI & IA IB & Print 16-bit word IA thru IB in INT*2 format & Y \\
\hline DI & IA IB & Display 16-bit word IA thru IB in INT*2 format & Y \\
\hline PIF & IA IB & Print 32-bit word IA thru IB in INT*4 format & Y \\
\hline DIF & IA IB & Display 32-bit word IA thru IB in INT*4 format & Y \\
\hline
\end{tabular}

Y: Command is, working

?: Command is not working or can not be checked because we have only one tape drive. 


\section{Appendix 3. MSU FTP Site Manual}

\section{FTP SITE_MANUAL}

\section{Last Edited Sat Jan 29 10:56:27 CST 1994}

SITE_MANUAL is a user manual of sorts for Mississippi State University Department of Physics and Astronomy Nuclear Group's anonymous ftp site. Feedback can be directed to cj@ph.msstate.edu,pgv1@ph.msstate.edu, or rbp@ph.msstate.edu.

INDEX

GAINING ACCESS.

RETRIEVING FILES.

DOWNLOADING FILES.

DIRECTORY HIERARCHY.

\section{GAINING ACCESS}

This section describes how to access this ftp site. Note that the information that is written to the screen may vary according to the version of the ftp software running on your computer.

your prompt $>$ ftp nucphy.ph.msstate.edu Connected to nucphy.ph.msstate.edu.

220 nucphy FTP server (SunOS 4.1) ready.

Name (nucphy.ph.msstate.edu:pgv1): anonymous

331 Guest login ok, send ident as password. 
Password:anonymous

230 Guest login ok, access restrictions apply.

$\mathrm{ftp}>\mathrm{ls}$

200 PORT command successful.

150 ASCII data connection for /bin/ls (130.18.152.62,1060) (0 bytes).

Incoming

SITE_MANUAL

bin

dev

etc

pub

usr

226 ASCII Transfer complete.

35 bytes received in 0.091 seconds $(0.38 \mathrm{Kbytes} / \mathrm{s})$

ftp>

\section{RETRIEVING FILES}

This section describes how to retrieve files from this site. After gaining access to this site (See above) decide what files are of intrest to you

(For more information see section DIRECTORY HIERARCHY) then change into the pub directory.

ftp $>c d$ pub

250 CWD command successful.

ftp $>$ ls

200 PORT command successful.

150 ASCII data connection for / bin/ls $(130.18 .152 .62,1284)$ (0 bytes).

\section{LISTINGS}

distributions

mirror

226 ASCII Transfer complete.

33 bytes received in 0.063 seconds $(0.51 \mathrm{Kbytes} / \mathrm{s})$ 
ftp>

Now change into the appropriate subdirector(ies/y) and retrieve the appropriate files with use of the get or mget commands. For on-line help type "help get" or "help mget" while in the ftp program. The "" are not necessary.

$\mathrm{ftp}>\mathrm{cd}$ distributions

250 CWD command successful.

ftp > cd subdirectory/subdirectory

250 CWD command successful.

ftp $>$ Is

README

file 1

fille2

file 3

226 ASCII Transfer complete.

47 bytes received in 0.067 seconds $(0.68 \mathrm{Kbytes} / \mathrm{s})$

ftp> get README

200 PORT command successful.

150 ASCII data connection for README (130.18.152.62,1287) (2171 bytes).

226 ASCII Transfer complete.

local: README remote: README

2225 bytes received in 0.048 seconds ( $46 \mathrm{Kbytes} / \mathrm{s}$ )

ftp $>$ quit

221 Goodbye.

your prompt>

\section{DOWNLOADING FILES}


This section describes how to upload software onto this anonymous ftp site.

After gaining access to this site (See section GAINING ACCESS) change into the Incoming directory. Only authorized users may upload files to this $\mathrm{ftp}$ site.

If you would line to get authorization to upload software that is consistent with the field of Nuclear Physics please e-mail cj@ph.msstate.edu or pgv1@ph.msstate.edu.

ftp>cd Incoming

250 CWD command successful.

$\mathrm{ftp}>\mathrm{ls}$

200 PORT command successful.

150 ASCII data connection for / bin/ls $(130.18 .152 .62,1061)$ ( 0 bytes).

Radware

SUNPAK

USORT

misc

226 ASCII Transfer complete.

30 bytes received in 0.1 seconds $(0.29 \mathrm{Kbytes} / \mathrm{s})$

$\mathrm{ftp}>$

Then change into the appropriate subdirectory and use the put or mput commands to upload the disired files. For on-line help type "help put" or "help mput" while in the ftp program. The "' are not necessary. The subdirectory should be one of the following, Radware, SUNPAK, USORT, or misc.

$\mathrm{ftp}>\mathrm{cd}$ subdirectory

250 CWD command successful.

ftp> put your_files

200 PORT command successful.

150 ASCII data connection for this_file $(130.18 .152 .62,1064)$.

226 ASCn Transfer complete.

$\mathrm{ftp}>$

For security reasons users will nor be allowed to read the Incoming 
subdirectories. However, this does not mean that your files were not uploaded. If the suspense of whether or not all was succesful e-mail either cj@ph.msstate.edu or pgv1@ph.msstate.edu and we will acknowledge the upload.

$\mathrm{ftp}>\mathrm{pwd}$

257 "/Incoming/subdirectory" is current directory.

$\mathrm{ftp}>\mathrm{ls}$

200 PORT command successful.

150 ASCII data connection for $/ \mathrm{bin} / \mathrm{s}(130.18 .152 .62,1063)$ ( 0 bytes).

. unreadable

226 ASCII Transfer complete.

14 bytes received in 0.073 seconds $(0.19 \mathrm{Kbytes} / \mathrm{s})$

ftp $>$ quit

221 Goodbye.

your prompt>

\section{DIRECTORY HIERARCHY}

This section describes the directory hierarchy and gives a description of the contents of each directory along with each file in the directory. SITE_MANUAL is this document which is a user manual for this site.

drwxr-xr-x 6 cj
-rw-r-r-- 1 pgv1


drwxrwxr-x 2 cj

drwxrwxr-x $4 \mathrm{cj}$

drwxrwxr-x $3 \mathrm{cj}$

bin:

systems directory

dev:

systems directory

etc:

systems directory

usr:

systems directory
512 Jul $61993 \mathrm{etc} /$

$512 \mathrm{Jan} 2714: 11 \mathrm{pub} /$

512 Jul 61993 usr/

Incoming:

total 4

drwxrwx-wx 2 cj

512 Jan 26 09:32 Radware/

drwxrwx-wx 2 cj

512 Jan 26 10:57 SUNPAKJ

drwxrwx-wx 2 cj

512 Jan 27 13:13 USORT/

drwxrwx-wx 2 cj

512 Jan 26 09:33 misc/

Incoming/Radware:

This directory is used exclusively for uploads pertaining to Radware.

Incoming/SUNPAK:

This directory is used exclusively for uploads pertaining to SUNPAK.

Incoming/USORT:

This directory is used exclusively for uploads pertaining to USORT.

Incoming/misc: 
This directory is used for uploads not pertaining to any of the above.

This directory contains a file called LISTINGS which is a listing 'ls - $-R^{2}$ ' of all of the files on this site at this level and lower. All downloading should be done from this directory and below.

pub:

total 3

$-r w-r w-r--1 \mathrm{cj}$ 853 Jan 20 08:32 LISTINGS

drwxrwxr-x 7 cj 512 Jan 27 13:13 distributions/

drwxr-xr-x 3 root 512 Jan 27 13:17 mirror/

This directory contains subdirectories that contian software that is distributed by or through the Nuclear Group's ftp site.

pub/distributions:

total 6

drwxrwar-x $2 \mathrm{cj}$

512 Sep 10 10:18 Radware/

drwxr-xr-x 2 cj

512 Sep 3 08:13 Sol_Dev_Con/

drwxr-xr-x 2 root

512 Jan 28 07:11 UHO.h/

drwxr-xr-x 3 root

512 Jan 27 13:13 USORT/

drwxr-xr-x 4 cj

512 Oct 7 07:35 development-iools/

drwxrwxr-x 2 cj

512 Jan $816: 45$ show/

This directory contains the latest version of David Radford and 
Randy MacLeod's high-spin data analysis software for Unix.

pub/distributions/Radware:

total 11131

-rw-rw-r-- 1 cj 4973921 Aug 31 09:32 Escl8rDemoDec.tar.Z

-rw-rw-r-- 1 cj 3805335 Aug 31 09:15 Escl8rDemoSun.tar.Z

-rw-rw-r-- $1 \mathrm{cj} \quad 2479$ Sep 10 10:18 README

-rw-rw-r-- 1 cj 1716545 Aug 31 09:03 RadwareAug93.tar.Z

-rw-rw-r-- 1 cj 412623 Aug 31 10:23 iccDEC.tar.Z

-rw-rw-r-- 1 cj 425314 Aug 31 10:38 iccSUN.tar.Z

This directory contains slides and CD materials from the technical workshops at the March 1993 Solaris Developer Conference.

pub/distributions/Sol_Dev_Con:

total 1457

-rw-r-r- - $1 \mathrm{cj} \quad 279$ Sep 3 08:11 README

-rW-r--r-- $1 \mathrm{cj} \quad 121035$ Sep 3 07:54 ddi.tar.Z

-rw-r--r-- 1 cj 897329 Sep $307: 57$ mt.tar.Z

-rw-r-r-- 1 cj 436763 Sep 3 07:54 xglpex.tar.Z

This directory contains the Universal Histogram Object (UHO) header file.

pub/distributions/UHO.h:

total 19

-rw-r--r-- 1 root 19169 Jan 28 07:11 uho.h 
This directory contains USORT software and related material. USORT is a UNIX based modular program for the sorting of gamma-ray spectroscopic data.

pub/distributions/USORT:

total 1

drwxr-xr-x $2 \mathrm{ftp} \quad 512 \operatorname{Jan} 27$ 13:13 usort1.1B_distribution_notes/

This directory contains USORT distribution notes.

pub/distributions/USORT/usort1.1B_distribution_notes:

total 58

-rw-r-r-- $1 \mathrm{ftp} \quad 3255 \mathrm{Jan} 26$ 19:50 ABOUT_USORT

-rw-r--r-- $1 \mathrm{ftp} \quad 592$ Jan 26 19:50 ACQUIRING_USORT

-rw-r-r-- $1 \mathrm{ftp} 9157$ Jan 26 19:50 USORT_COMMANDS

-rw-r--r-- $1 \mathrm{ftp} \quad 2768$ Jan 26 19:50 USORT_DISCLAIMER

-rw-r--r-- $1 \mathrm{ftp} \quad 5678$ Jan 26 19:50 USORT_HISTORY

-rW-r-r-r $1 \mathrm{ftp} \quad$ 10251 Jan 26 19:50 USORT_INSTALLATION

-rw-r--r-- $1 \mathrm{ftp} \quad 23865 \mathrm{Jan} 26$ 19:50 USORT_OVERVIEW

This directory contains ToolTalk programs and utilities for programming with Sun's ToolTalk.

pub/distributions/development-tools:

total 446

drwxr-xr-x 2 cj 512 Oct 7 07:31 .cap/

-rw-r--r-- $1 \mathrm{cj} \quad 265$ Oct 7 07:31 INDEX

-rw-r--r-- $1 \mathrm{cj} \quad 129$ Oct 7 07:31 README

drwxr-xr-x 3 cj $\quad 512$ Oct 7 07:32 ToolTalk/ 
-rw-r--r-- 1 cj 924 Oct 7 07:31 appmap.iafa

-rw-r--r-- 1 cj $\quad 81920$ Oct 7 07:31 appmap.tar

-rw-r--r-- 1 cj 1014 Oct 7 07:31 solaris2_sample_drivers_june93.iafa

-rw-r--r-- 1 cj 358201 Oct 7 07:31 solaris2_sample_drivers_june93.tar.Z

This directory contains ToolTalk programs and utilities for programming with Sun's ToolTalk.

pub/distributions/development-tools/.cap:

total 3

-rw-r--r-- 1 cj $\quad 75$ Oct 7 07:31 ToolTalk

-rw-r-r-r- 1 cj $\quad 81$ Oct 7 07:31 appmap.tar

-rw-r--r-- 1 cj 96 Oct 7 07:31 solaris2_sample_drivers_june93.tar.Z

This directory contains ToolTalk programs and utilities for programming with Sun's ToolTalk.

pub/distributions/development-tools/TcolTalk:

total 194

$$
\begin{array}{lc}
\text { drwxr-xr-x } 2 \text { cj } & 512 \text { Oct } 7 \text { 07:32 .cap/ } \\
\text {-rw-r-r-- } 1 \text { cj } & 244 \text { Oct } 7 \text { 07:31 INDEX } \\
\text {-rw-r-r-- } 1 \text { cj } & 134 \text { Oct } 7 \text { 07:31 README } \\
\text {-rw-r--r-- } 1 \text { cj } & 750 \text { Oct } 7 \text { 07:32 TT-c++.iafa } \\
\text {-rw-r-r-- } 1 \text { cj } & 99107 \text { Oct } 7 \text { 07:32 TT-c++.tar.Z } \\
\text {-rw-r-r-- } 1 \text { cj } & 659 \text { Oct } 7 \text { 07:32 ttsnoop.iafa } \\
\text {-rw-r-r-- } 1 \text { cj } & 78131 \text { Oct 7 07:32 ttsnoop.tar.Z }
\end{array}
$$


pub/distributions/development-tools/ToolTalk/cap:

total 0

This directory contains source code for the UHO file format.

The object of show is to demonstrate the use of a universal histogram data storage format. Show executes a series of functions that manipulate the histogram record in memory. The idea in separating all the record handling functions was to give other programmers the option of using our record handling routines without necessarily using our program. All of the universal histogram handling routines are contained in the directory src/uho of the release.

pub/distributions/show:

total 389

-rw-r--r-- $1 \mathrm{cj}$
-rw-r--r-- $1 \mathrm{cj}$
-rw-r-r-- $1 \mathrm{cj}$
2171 Jan 8 8 16:45 README
-rw-r--r-- $1 \mathrm{cj}$

This directory contains mirrored software from other anonymous ftp sites.

pub/mirror:

total 1

drwxr-xr-x 5 cj 512 Jan 27 13:17 Linux/ 
This directory contains Linux software and documentation which is a UNIX-like operating the runs on a PC. Slackware is mirrored from sunsite.unc.edu and contains the full operating system, gnu compilers and Xfree 86 release.

pub/mirror/Linux:

total 4

drwxr-xr-x 3 cj $\quad 512$ Nov 9 15:38 linux.docs/

d.wxr-xr-x 2 cj $\quad 512$ Nov $918: 15$ misc/

drwxr-xr-x 45 cj 1536 Nov $916: 38$ slackware/

This directory contains linux documentations.

pub/mirror/Linux/linux.docs:

total 307

\begin{tabular}{|c|c|}
\hline$-r w-r--r--1 c j$ & 11253 Aug 22 17:2.8 LILO-FAQ \\
\hline$-r w-r-r--1 c j$ & 43324 Aug 22 18:41 NET-2-FAQ \\
\hline$-r w-r--r--1 c j$ & 38237 Jul 211993 faq_11.p1 \\
\hline$-r w-r--r--1 c j$ & 56179 Jul 211993 faq_11.p2 \\
\hline$-r w-r-r--1 c j$ & 33478 Jul 211993 faq_11.p3 \\
\hline$-r w-r--r--1 c j$ & 29999 Jul 211993 faq_11.p4 \\
\hline$-r W-r-r--1 c j$ & 42286 Jul 211993 faq_11.p5 \\
\hline$-r w-r--r--1 c j$ & 48264 Jul 211993 faq_11.p6 \\
\hline$-r w-r-r--1 c j$ & 5230 Jul 211993 faq_11.toc \\
\hline drwxr-xr-x 2 cj & 512 Jun $211993 \mathrm{ps} /$ \\
\hline
\end{tabular}

This directory contains linux documentations in the postscript format.

pub/mirror/Linux/linux.docs/ps: 
total 1472

-rw-r--r-- 1 cj 739505 Jun 211993 faq_1.ps

-rw-r--r-- 1 cj 742550 Jun 211993 faq_2.ps

This directory contains miscellaneous linux files.

pub/mirror/Linux/misc:

total 2154

-rw-r--r-- 1 cj 120822 Nov 9 18:15 distribution-HOWTO

-rw-r--r-- 1 cj 315933 Nov 9 16:46 dungeon-src.tar.Z

-rw-r--r-- 1 cj 277478 Nov 9 16:46 gnuchess-src.tar.Z

-rw-r--r-- 1 cj 1337669 Nov 9 16:47 linux-0.99.13.tar.Z

-rw-r--r-- 1 cj 13052 Oct $710: 31$ rawrite.exe

-rw-r--r-- $1 \mathrm{cj} \quad 2835$ Nov 9 16:36 snd-driv-2.0.README

-rw-r--r-- 1 cj 91484 Nov 9 16:37 snd-util-2.0.tar.gz

The slackware package contains the linux kernel and utilities, gnu compilers and utilities, the Xfree86 windows package and an automated installation process.

pub/mirror/Linux/slackware:

total 8690

\begin{tabular}{|c|c|}
\hline$-r w-r--r--1 c j$ & 1768 Nov 9 13:47.desc.txt \\
\hline$-r W-r--r--1 c j$ & 9655 Nov 9 13:47 00index.txt \\
\hline$-r w-r--r--1 c j$ & 4475 Nov 9 13:47 ANNOUNCE.1.0.2 \\
\hline$-r w-r--r--1 c j$ & 27 Nov 9 13:47 ANNOUNCE.1.0.4 \\
\hline$-r w-r--r--1 c j$ & 27 Nov 9 13:47 ANNOUNCE.1.0.5 \\
\hline$-r W-r--r--1 c j$ & 4741 Nov 9 13:47 ANNOUNCE.1.1.0 \\
\hline$-r w-r--r--1 c j$ & 19329 Nov 9 13:47 FILE_LIST \\
\hline$-r W-r--r--1$ & 6126 Nov 9 13:47 OldChangeLog \\
\hline
\end{tabular}




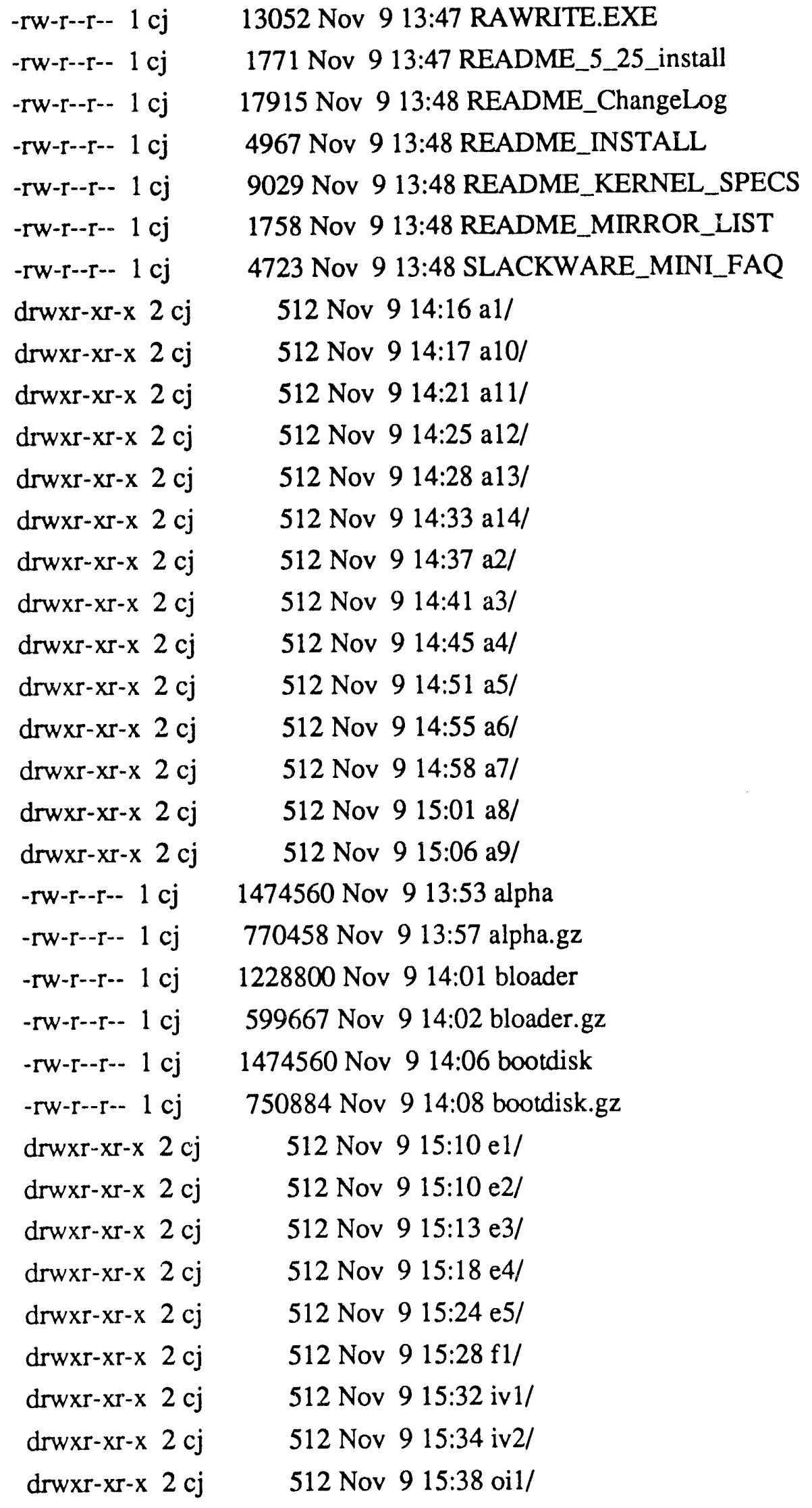




\begin{tabular}{|c|c|}
\hline drwxr-xr-x 2 cj & 512 Nov 9 15:41 oi $2 /$ \\
\hline drwxr-xr-x $2 c j$ & 512 Nov 9 15:42 oi3/ \\
\hline$w x r-x r-x \quad 2 c j$ & 512 Nov $915: 47$ oop $1 /$ \\
\hline$w x r-x r-x 2 c j$ & 512 Nov 9 15:48 q1/ \\
\hline Irwxr-xr-x $2 \mathrm{cj}$ & 512 Nov 9 15:49 q2/ \\
\hline$r w-r--r--1 c j$ & 1474560 Nov $914: 11$ rescue \\
\hline rw-r-r-r-- $1 \mathrm{cj}$ & 890063 Nov 9 14:13 rescue.gz \\
\hline lrwxr-xr-x $2 c j$ & 512 Nov $915: 52$ scripts/ \\
\hline lrwxr-xr-x $2 c j$ & 512 Nov $915: 53$ tcl $1 /$ \\
\hline rwxr-xr-x $2 \mathrm{cj}$ & 512 Nov $915: 58 \times 1 /$ \\
\hline Irwxr-xr-x $2 \mathrm{cj}$ & 512 Nov $916: 01 \times 2 /$ \\
\hline $\mathrm{w} w \mathrm{xr}-\mathrm{xr}-\mathrm{x} 2 \mathrm{cj}$ & 512 Nov $916: 04 \times 3 /$ \\
\hline drwxr-xr-x 2 cj & 512 Nov $916: 07 \times 4 /$ \\
\hline drwxr-xr-x 2 cj & 512 Nov $916: 10 \times 5 /$ \\
\hline drwxr-xr-x $2 \mathrm{cj}$ & 512 Nov 9 16:13 xap1/ \\
\hline drwxr-xr-x 2 cj & 512 Nov 9 16:14 хар2/ \\
\hline drwxr-xr-x $2 c j$ & 512 Nov $916: 17 \mathrm{xd} 1 /$ \\
\hline drwxr-xr-x $2 c j$ & 512 Nov $916: 20 \mathrm{xd} 2 /$ \\
\hline drwxr-xr-x $2 c j$ & 512 Nov $916: 23 \mathrm{xd} 3 /$ \\
\hline drwxr-xr-x 2 cj & 512 Nov $916: 26$ xv1/ \\
\hline$w x r-x r-x 2 c j$ & 512 Nov $916: 29$ xv2/ \\
\hline$w x r-x r-x \quad 2 c j$ & 512 Nov $916: 31$ yl/ \\
\hline
\end{tabular}

pub/mirror/Linux/slackware/a1:

total 1240

\begin{tabular}{|c|c|}
\hline$-\mathrm{rw}-\mathrm{r}-\mathrm{-r}-\mathrm{-} 1 \mathrm{cj}$ & 312 Nov 9 14:13 .desc.txt \\
\hline$-r w-r-r--1 c j$ & 312 Nov 9 14:13 00index.txt \\
\hline$-r w-r-r--1 c j$ & 401086 Nov $914: 14$ base.tgz \\
\hline$-r w-r-r--1 c j$ & 3293 Nov 9 14:14 devs.tgz \\
\hline$-r w-r-r--1 c j$ & 1509 Nov 9 14:14 diska 1 \\
\hline$-r w-r-r--1 c j$ & 23788 Nov 9 14:15 etc.tgz \\
\hline$-r w-r--r--1 c j$ & 258981 Nov $914: 15$ idekern.tgz \\
\hline$-r w-r-r--1 c j$ & 45199 Nov 9 14:15 lilo.tgz \\
\hline
\end{tabular}


-rw-r--r-- 1 cj 294043 Nov 9 14:15 scsikern.tgz

-rw-r--r-- 1 cj 166841 Nov 9 14:16 shadow.tgz

-rw-r--r-- 1 cj 8566 Nov 9 14:16 tagfile

-rW-r-r-- $1 \mathrm{cj} \quad 8566$ Nov 9 14:16 tagfile.org

-rw-r--r-- $1 \mathrm{cj} \quad 8513$ Nov 9 14:16 tagfile.pat

pub/mirror/Linux/slackware/a10:

total 1323

-rw-r--r-- 1 cj 104 Nov 9 14:16 desc.txt

-rw-r--r-- $1 \mathrm{cj} \quad 104$ Nov 9 14:16 00index.txt

-rW-r--r-- 1 cj 264 Nov 9 14:16 diska10

-rW-r-r-- 1 cj 533570 Nov $914: 17$ gcc245.tgz

-rW-r--r-- 1 cj 787416 Nov 9 14:19 gxx245.tgz

pub/mirror/Linux/slackware/a11:

total 1222

-rw-r--r-- 1 cj 208 Nov 9 14:19 desc.txt

-rw-r--r-- 1 cj 208 Nov 9 14:19 00index.txt

-rw-r--r-- 1 cj 449 Nov 9 14:19 diska11

-rw-r-r-r- 1 cj 490266 Nov 9 14:20 gdb.tgz

-rw-r--r-- 1 cj 2830 Nov 9 14:20 gonzo.tgz

-rw-r-r-- $1 \mathrm{cj} \quad 3063$ Nov $914: 20$ gp9600.tgz

-rW-r-r-- 1 cj 62037 Nov 9 14:20 ksh.tgz

-rW-r-r-r- $1 \mathrm{cj} \quad 368203$ Nov 9 14:21 lib444.tgz

-rw-r-r-- 1 cj 288530 Nov 9 14:23 p2c.tgz

pub/mirror/Linux/slackware/a12: 
total 1224

\begin{tabular}{|c|c|}
\hline$-r w-r--r--1 c j$ & 208 Nov 9 14:23, desc.txt \\
\hline$-r w-r--r--1 c j$ & 208 Nov 9 14:23 00index.txt \\
\hline$-r w-r--r--1 c j$ & 46244 Nov 9 14:23 dip.tgz \\
\hline$-r w-r--r--1 c j$ & 895 Nov $914: 23$ diska 12 \\
\hline$-r w-r--r--1 c j$ & 281828 Nov $914: 24$ english.tgz \\
\hline$-r w-r--r--1 c j$ & 184382 Nov $914: 24$ f2c.tgz \\
\hline$-r w-r--r--1 c j$ & 63942 Nov 9 14:24 lxpatch.tgz \\
\hline$-r w-r--r--1 c j$ & 114149 Nov $914: 25$ man2.tgz \\
\hline$r w-r--r--1 c j$ & 513682 Nov $914: 26$ objc $245 . \operatorname{tgz}$ \\
\hline
\end{tabular}

pub/mirror/Linux/slackware/a13:

total 1263

\begin{tabular}{|c|c|}
\hline$-r w-r--r--1 c j$ & 234 Nov 9 14:26 .desc.txt \\
\hline$-r W-r--r--1 c j$ & 234 Nov 9 14:26 00index.txt \\
\hline$-r w-r--r--1 c j$ & 65867 Nov 9 14:26 butils $10 . \operatorname{tgz}$ \\
\hline$-r w-r--r--1 c j$ & 1805 Nov 9 14:26 diska 13 \\
\hline$-r w-r--r--1 c j$ & 122822 Nov 9 14:27 man3.tgz \\
\hline$-r w-r--r--1 c j$ & 40343 Nov $914: 27$ manpgs.tgz \\
\hline$-r w-r--r--1 c j$ & 268972 Nov $914: 27$ pine3051.tgz \\
\hline$-r w-r--r--1 c j$ & 661009 Nov 9 14:28 smail.tgz \\
\hline$-r w-r--r--1 c j$ & 44580 Nov 9 14:28 sound.tgz \\
\hline$-r w-r--r--1 c j$ & 54365 Nov $914: 29 \operatorname{tar} 1112 . \operatorname{tgz}$ \\
\hline
\end{tabular}

pub/mirror/Linux/slackware/a14:

total 1420

$\begin{array}{lr}\text {-rw-r--r-- } 1 \mathrm{cj} & 130 \text { Nov } 914: 29 \text {.desc.txt } \\ \text {-rw-r--r-- } 1 \mathrm{cj} & 130 \text { Nov } 914: 2900 \text { index.txt } \\ \text {-rw-r--r-- } 1 \mathrm{cj} & 885 \text { Nov } 914: 29 \text { diska14 } \\ \text {-rw-r--r-- } 1 \mathrm{cj} & 784853 \text { Nov } 914: 31 \text { extra444.tgz }\end{array}$


-rw-r--r-- 1 cj 642595 Nov $914: 33$ groff.tgz

-rw-r--r-- $1 \mathrm{cj} \quad 154$ Nov $914: 33$ install.end

pub/mirror/Linux/slackware/a2:

total 1425

-rw-r--r-- 1 cj 338 Nov $914: 33$ desc.txt

-rw-r--r-- 1 cj 338 Nov 9 14:33 00index.txt

-rw-r--r-- 1 cj 649163 Nov $914: 34$ bin.tgz

-rw-r--r-- 1 cj 1038 Nov $914: 34$ diska2

-rw-r--r-- 1 cj 46921 Nov 9 14:34 e2fsbn.tgz

-rw-r--r-- 1 cj 29081 Nov 9 14:34 getty.tgz

-rw-r--r-- 1 cj 38453 Nov 9 14:34 gzip124.tgz

-rw-r--r-- 1 cj 68763 Nov $914: 35$ lpr.tgz

-rw-r--r-- 1 cj 45911 Nov $914: 35$ make368.tgz

-rw-r--r-- 1 cj 39490 Nov $914: 35$ ps.tgz

-rw-r--r-- 1 cj 136626 Nov $914: 35$ shlibs.tgz

-rw-r--r-- 1 cj 172893 Nov 9 14:36 usrbin4.tgz

-rw-r--r-- 1 cj 143186 Nov 9 14:36 util.tgz

-rw-r--r-- 1 cj 28267 Nov $914: 37$ zoneinfo.tgz

pub/mirror/Linux/slackware/a3:

total 1345

-rw-r--r-- 1 cj $\quad 312$ Nov $914: 37$.desc.txt

-rw-r--r-- 1 cj 312 Nov 9 14:37 00index.txt

-rW-r--r-- 1 cj 1478 Nov $914: 37$ diska3

-rW-r-r-- 1 cj 199405 Nov 9 14:38 dos049.tgz

-rw-r--r-- 1 cj 53232 Nov $914: 38$ pmake44b.tgz

-rw-r--r-- 1 cj 167969 Nov $914: 39$ pmake44s.tgz

-rw-r--r-- 1 cj 166504 Nov $914: 39$ rcs56a.tgz

-rw-r--r-- 1 cj 90810 Nov $914: 40$ sc.tgz 


$$
\begin{array}{ll}
\text {-rw-r--r-- } 1 \text { cj } & 18973 \text { Nov } 9 \text { 14:40 select15.tgz } \\
\text {-rw-r--r-- } 1 \text { cj } & \text { 20776 Nov 9 14:40 sysvinit.tgz } \\
\text {-rw-r--r-- } 1 \text { cj } & 148751 \text { Nov 9 14:40 tcsh604.tgz } \\
\text {-rw-r--r-- } 1 \text { cj } & 40199 \text { Nov } 914: 40 \text { term108x.tgz } \\
\text {-rw-r--r-- } 1 \text { cj } & 399184 \text { Nov 9 14:42 uucp104.tgz }
\end{array}
$$

pub/mirror/Linux/slackware/a4:

total 1328

$$
\begin{array}{lc}
\text {-rw-r-r-- } 1 \text { cj } & 234 \text { Nov } 9 \text { 14:42 desc.txt } \\
\text {-rw-r--r-- } 1 \text { cj } & 234 \text { Nov } 9 \text { 14:42 00index.txt } \\
\text {-rw-r--r-- } 1 \text { cj } & 146157 \text { Nov 9 14:42 cnews.tgz } \\
\text {-rw-r--r-- } 1 \text { cj } & 645 \text { Nov } 9 \text { 14:42 diska4 } \\
\text {-rw-r--r-- } 1 \text { cj } & 53055 \text { Nov 9 14:43 gettys.tgz } \\
\text {-rw-r--r-- } 1 \text { cj } & 83249 \text { Nov 9 14:43 joe.tgz } \\
\text {-rw-r--r-- } 1 \text { cj } & 44454 \text { Nov 9 14:43 keytbls.tgz } \\
\text {-rw-r--r-- } 1 \text { cj } & 482492 \text { Nov 9 14:45 man.tgz } \\
\text {-rw-r--r-- } 1 \text { cj } & \text { 26739 Nov 9 14:45 national.tgz } \\
\text {-rw-r--r-- } 1 \text { cj } & 490119 \text { Nov 9 14:46 tcpip.tgz }
\end{array}
$$

pub/mirror/Linux/slackware/a5:

total 1383

$$
\begin{array}{ll}
\text {-rw-r-r-- } 1 \text { cj } & 416 \text { Nov 9 14:46 desc.txt } \\
\text {-rw-r--r-- } 1 \text { cj } & \text { 416 Nov 9 14:46 00index.txt } \\
\text {-rw-r--r-- } 1 \text { cj } & 136184 \text { Nov 9 14:47 bin4.tgz } \\
\text {-rw-r--r-- } 1 \text { cj } & 221253 \text { Nov 9 14:48 comms.tgz } \\
\text {-rw-r--r-- } 1 \text { cj } & \text { 28025 Nov 9 14:48 cpio23.tgz } \\
\text {-rw-r--r-- } 1 \text { cj } & \text { 29085 Nov 9 14:48 deliver.tgz } \\
\text {-rw-r--r-- } 1 \text { cj } & 41043 \text { Nov 9 14:48 diff24.tgz } \\
\text {-rw-r--r-- } 1 \text { cj } & \text { 2330 Nov 9 14:48 diska5 } \\
\text {-rw-r--r-- } 1 \text { cj } & \text { 32318 Nov 9 14:48 e2fssrc.tgz }
\end{array}
$$




$$
\begin{aligned}
& \text {-rw-r--r-- } 1 \text { cj } 71124 \text { Nov } 914: 48 \text { elfabi.tgz } \\
& \text {-rw-r--r-- } 1 \mathrm{cj} \quad 126679 \text { Nov } 914.49 \text { jove4147.tgz } \\
& \text {-rw-r--r-- } 1 \mathrm{cj} \quad 4931 \text { Nov } 914: 49 \mathrm{mt} . t g z \\
& \text {-rw-r--r-- } 1 \text { cj } 216961 \text { Nov } 914: 49 \text { nn6418.tgz } \\
& \text {-rw-r--r-- } 1 \text { cj } 122880 \text { Nov } 914: 50 \text { nn6418m.tar } \\
& \text {-rw-r--r-- } 1 \text { cj } 177330 \text { Nov } 914: 51 \text { svgalib8.tgz } \\
& \text {-rW-r--r-- } 1 \text { cj } 93932 \text { Nov } 914: 51 \text { term108s.tgz } \\
& \text {-rW-r--r-- } 1 \text { cj } 33191 \text { Nov } 914: 51 \text { tracrout.tgz }
\end{aligned}
$$

pub/mirror/Linux/slackware/a6:

total 1196

$$
\begin{aligned}
& \text {-rw-r-r-- } 1 \mathrm{cj} \quad 182 \text { Nov } 914: 51 \text { desc.txt } \\
& \text {-rw-r--r-- } 1 \mathrm{cj} \quad 182 \text { Nov } 914: 51 \text { 00index.txt } \\
& \text {-rW-r-r-- } 1 \text { cj } 138029 \text { Nov } 914: 51 \text { binutils.tgz } \\
& \text {-rW-r--r-- } 1 \text { cj } 471733 \text { Nov } 914: 53 \text { clisp.tgz } \\
& \text {-rw-r--r-- } 1 \mathrm{cj} \quad 880 \text { Nov } 914: 53 \text { diska6 } \\
& \text {-rw-r--r-- } 1 \text { cj } 495624 \text { Nov } 914: 55 \text { perl.tgz } \\
& \text {-rw-r--r-- } 1 \text { cj } 52272 \text { Nov } 9 \text { 14:55 quota.tgz } \\
& \text {-rw-r--r-- } 1 \text { cj } 29242 \text { Nov } 914: 55 \text { syslogd.tgz }
\end{aligned}
$$

pub/mirror/Linux/slackware/a7:

total 1267

$$
\begin{array}{lc}
\text {-rw-r--r-- } 1 \mathrm{cj} & 130 \text { Nov } 914: 55 \text { desc.txt } \\
\text {-rw-r--r-- } 1 \mathrm{cj} & 130 \text { Nov } 914: 55 \text { 00index.txt } \\
\text {-rw-r--r-- } 1 \mathrm{cj} & 388 \text { Nov } 914: 55 \text { diska7 } \\
\text {-rw-r--r-- } 1 \mathrm{cj} & 25739^{r} \text { Nov } 914: 56 \text { elm2421.tgz } \\
\text {-rw-r--r-- } 1 \mathrm{cj} & 880157 \text { Nov } 914: 58 \text { lx99p13.tgz } \\
\text {-rw-r--r-- } 1 \mathrm{cj} & 118555 \text { Nov } 914: 59 \text { tin12p2.tgz }
\end{array}
$$


pub/mirror/Linux/slackware/a8:

total 1408

$\begin{array}{lc}\text {-rw-r--r-- } 1 \text { cj } & 182 \text { Nov } 9 \text { 14:59 .desc.txt } \\ \text {-rw-r--r-- } 1 \text { cj } & 182 \text { Nov } 9 \text { 14:59 00index.txt } \\ \text {-rw-r--r-- } 1 \text { cj } & 1129 \text { Nov 9 14:59 diska8 } \\ \text {-rw-r--r-- } 1 \text { cj } & 488105 \text { Nov 9 14:59 ghostscr.tgz } \\ \text {-rw-r--r-- } 1 \text { cj } & 497255 \text { Nov 9 15:00 gsfonts } 1 . t g z \\ \text {-rw-r--r-- } 1 \text { cj } & 44443 \text { Nov 9 15:00 mailx.tgz } \\ \text {-rw-r--r-- } 1 \text { cj } & 196078 \text { Nov 9 15:01 ncurses.tgz } \\ \text {-rw-r--r-- } 1 \text { cj } & 164083 \text { Nov 9 15:02 trn25.tgz }\end{array}$

pub/mirror/Linux/slackware/a9:

total 1358

\begin{tabular}{|c|c|}
\hline$-r w-r-r--1 c j$ & 130 Nov 9 15:02 .desc.txt \\
\hline$-r w-r-r-r-1 c j$ & 130 Nov 9 15:02 00index.txt \\
\hline$-r w-r-r--1 c j$ & 35703 Nov 9 15:02 bc.tgz \\
\hline$-r w-r-r--1 c j$ & 240 Nov 9 15:02 diska 9 \\
\hline$-r w-r-r--1 c j$ & 1195121 Nov 9 15:06 gsfonts2.tgz \\
\hline$-r w-r--r--1 c j$ & 134118 Nov $915: 06$ inc $444 . \operatorname{tgz}$ \\
\hline
\end{tabular}

pub/mirror/Linux/slackware/el:

total 1015

$$
\begin{aligned}
& \text {-rw-r--r-- } 1 \text { cj } 130 \text { Nov } 9 \text { 15:06 desc.txt } \\
& \text {-rw-r-r-- } 1 \mathrm{cj} \quad 130 \text { Nov } 9 \text { 15:06 00index.txt } \\
& \text {-rw-r--r-- } 1 \text { cj } 355 \text { Nov 9 15:06 diske1 } \\
& \text {-rW-r--r-- } 1 \text { cj } 1018813 \text { Nov } 9 \text { 15:10 ebin19a.tgz } \\
& \text {-rw-r--r-- } 1 \mathrm{cj} \quad 1941 \text { Nov } 9 \text { 15:10 tagfile } \\
& \text {-rW-r-r-r- } 1 \text { cj } 1941 \text { Nov 9 15:10 tagfile.org }
\end{aligned}
$$


pub/mirror/Linux/slackware/e2:

total 939

-rw-r--r-- 1 cj $\quad 78$ Nov 9 15:10 desc.txt

-rW-r--r-- 1 cj 78 Nov 9 15:10 00index.txt

-rw-r-r-- 1 cj 130 Nov $915: 10$ diske2

-rw-r--r-- 1 cj 950190 Nov 9 15:13 e19misc.tgz

pub/mirror/Linux/slackware/e3:

total 1243

-rW-r--r-- 1 cj 78 Nov 9 15:13 .desc.txt

-rW-r--r-- 1 cj 78 Nov 9 15:13 00index.txt

-rW-r--r-- 1 cj 281 Nov 9 15:13 diske3

-rw-r--r-- 1 cj 1254727 Nov 9 15:18 e19elc.tgz

pub/mirror/Linux/slackware/e4:

total 1051

-rw-r-r-r- 1 cj $\quad 78$ Nov 9 15:18 desc.txt

-rw-r-r-- $1 \mathrm{cj} \quad 78$ Nov 9 15:18 00index.txt

-rW-r--r-- $1 \mathrm{cj} \quad 161$ Nov 9 15:18 diske4

-rW-r--r-- 1 cj 1061412 Nov 9 15:21 e19els1.tgz

pub/mirror/Linux/slackware/e5:

total 796 


$$
\begin{array}{lc}
\text {-rw-r--r-- } 1 \text { cj } & 104 \text { Nov } 9 \text { 15:21 desc.txt } \\
\text {-rw-r--r-- } 1 \text { cj } & 104 \text { Nov } 915: 21 \text { 00index.txt } \\
\text {-rw-r--r-- } 1 \text { cj } & 161 \text { Nov } 915: 21 \text { diske5 } \\
\text {-rw-r--r-- } 1 \text { cj } & 802082 \text { Nov } 915: 24 \text { e19els2.tgz } \\
\text {-rw-r--r-- } 1 \text { cj } & 43 \text { Nov } 915: 24 \text { install.end }
\end{array}
$$

pub/mirror/Linux/slackware/f1:

total 990

$$
\begin{aligned}
& \text {-rw-r--r-- } 1 \mathrm{cj} \quad 260 \text { Nov } 9 \text { 15:24 desc.txt } \\
& \text {-rw-r--r-- } 1 \text { cj } 260 \text { Nov } 9 \text { 15:24 00index.txt } \\
& \text {-rw-r--r-- } 1 \text { cj } 647 \text { Nov } 9 \text { 15:24 diskfl } \\
& \text {-rw-r--r-- } 1 \mathrm{cj} \quad 36 \text { Nov } 9 \text { 15:24 install.end } \\
& \text {-rw-r--r-- } 1 \text { cj } 3490 \text { Nov } 9 \text { 15:25 install.txt } \\
& \text {-rw-r--r-- } 1 \text { cj } 2674 \text { Nov } 9 \text { 15:25 makeflop } \\
& \text {-rw-r--r-- } 1 \text { cj } 982932 \text { Nov } 9 \text { 15:28 manyfaqs.tgz } \\
& \text {-rw-r--r-- } 1 \mathrm{cj} \quad 1771 \text { Nov } 9 \text { 15:28 read5_25.txt } \\
& \text {-rw-r--r-- } 1 \text { cj } 4723 \text { Nov } 9 \text { 15:28 slakware.faq } \\
& \text {-rw-r--r-- } 1 \text { cj } 1676 \text { Nov } 915: 28 \text { tagfile } \\
& \text {-rw-r--r-- } 1 \mathrm{cj} \quad 1676 \text { Nov } 915: 28 \text { tagfile.org }
\end{aligned}
$$

pub/mirror/Linux/slackware/iv1:

total 1319

$$
\begin{aligned}
& \text {-rw-r-.r-- } 1 \text { cj } 156 \text { Nov } 9 \text { 15:28 .desc.txt } \\
& \text {-rw-r--r-- } 1 \mathrm{cj} \quad 156 \text { Nov } 915: 28 \text { 00index.txt } \\
& \text {-rW-5--r-- } 1 \mathrm{cj} \quad 118 \text { Nov } 915: 28 \text { diskiv } 1 \\
& \text {-rw-r--r-- } 1 \text { cj } 160765 \text { Nov } 9 \text { 15:28 ivincs.tgz } \\
& \text {-rw-r--r-- } 1 \text { cj } 1161472 \text { Nov } 9 \text { 15:32 ivlibs31.tgz } \\
& \text {-rw-r-r-- } 1 \text { cj } 1849 \text { Nov } 915: 32 \text { tagfile } \\
& \text {-rw-r--r-- } 1 \mathrm{cj} \quad 1849 \text { Nov } 9 \text { 15:32 tagfile.org }
\end{aligned}
$$


pub/mirror/Linux/slackware/iv2:

total 836

$\begin{array}{lc}\text {-rw-r-r-- } 1 \text { cj } & 130 \text { Nov } 915: 32 \text {.desc.txt } \\ \text {-rw-r--r-- } 1 \text { cj } & 130 \text { Nov } 915: 32 \text { 00index.txt } \\ \text {-rw-r--r-- } 1 \text { cj } & 124 \text { Nov } 915: 32 \text { diskiv2 } \\ \text {-rw-r-r-- } 1 \text { cj } & 351458 \text { Nov } 915: 33 \text { doc31.tgz } \\ \text {-rw-r-r-- } 1 \text { cj } & 475731 \text { Nov } 915: 34 \text { idraw.tgz } \\ \text {-rw-r--r-- } 1 \text { cj } & 14 \text { Nov } 915: 34 \text { install.end }\end{array}$

pub/mirror/Linux/slackware/oil:

total 1290

\begin{tabular}{|c|c|}
\hline$-r w-r--r--1 c j$ & 182 Nov 9 15:34 desc.txt \\
\hline$-r w-r-r--1 c j$ & 182 Nov 9 15:34 00index.txt \\
\hline -rw-r--r-- $1 \mathrm{cj}$ & 7398 Nov $915: 34$ aletter.txt \\
\hline$-r w-r-r--1 c j$ & 1886 Nov $915: 34$ copying \\
\hline$-r w-r-r--1 ~ c j$ & 243 Nov 9 15:34 diskoil \\
\hline$-r w-r--r--1 c j$ & 225000 Nov 9 15:35 oiinc.tgz \\
\hline$-r w-r-r--1 c j$ & 1029908 Nov $915: 38$ oilib.tgz \\
\hline$-r w-r-r--1 c j$ & 29589 Nov 9 15:38 oimisc.tgz \\
\hline
\end{tabular}




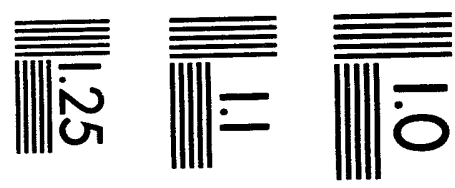

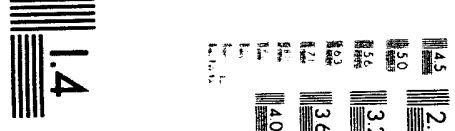

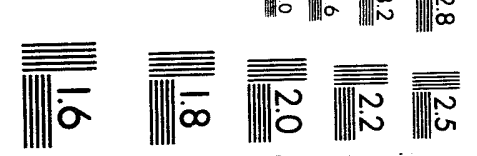



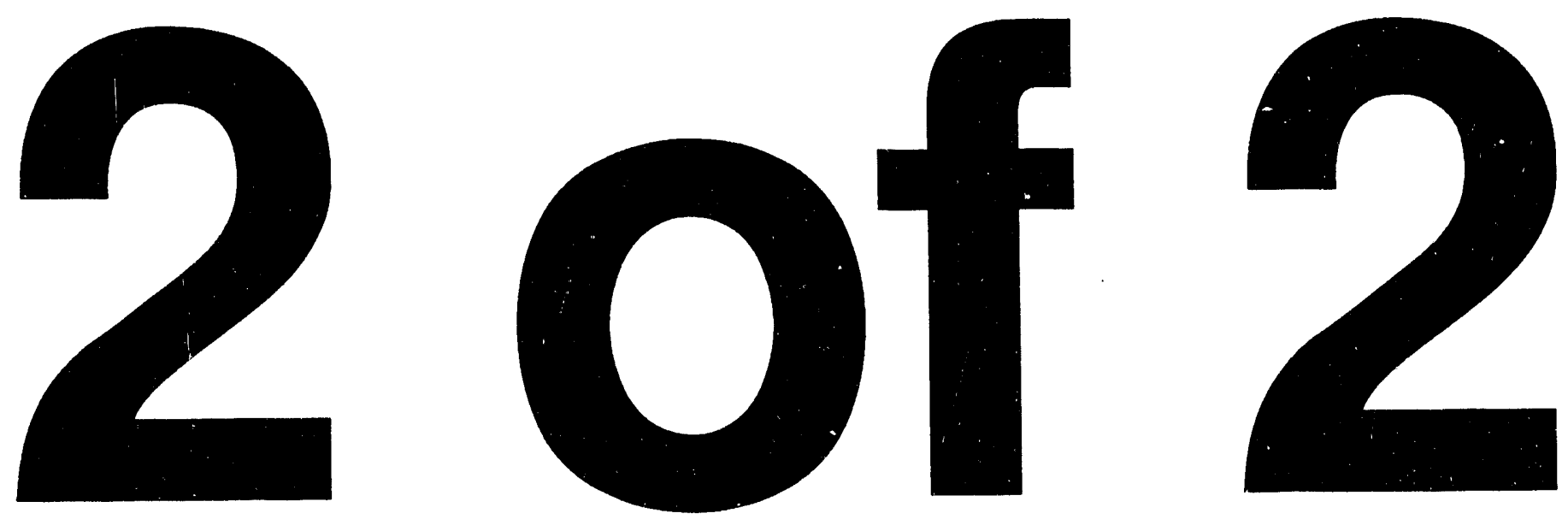
pub/mirror/Linux/slackware/oi2:

total 861

\begin{tabular}{|c|c|}
\hline -rw-r--r-- $1 \mathrm{cj}$ & 182 Nov 9 15:38 .desc.txt \\
\hline$-r w-r-r--1 \mathrm{cj}$ & 182 Nov 9 15:38 00index.txt \\
\hline -rw-r-r-r- $1 \mathrm{cj}$ & 1886 Nov $915: 38$ copying \\
\hline$-r w-r--r--1 c j$ & 324 Nov 9 15:38 diskoi2 \\
\hline$-r w-r--r--1 c j$ & 135913 Nov 9 15:39 oidemos.tgz \\
\hline$-\mathrm{rw}-\mathrm{r}-\mathrm{-r}-\mathrm{-} 1 \mathrm{cj}$ & 207893 Nov 9 15:40 oidoc.tgz \\
\hline$-r w-r--r--1 c j$ & 165860 Nov $915: 41$ oiman.tgz \\
\hline$-r w-r--r--1 c j$ & 317779 Nov 9 15:41 uib.tgz \\
\hline
\end{tabular}

pub/mirror/Linux/slackware/oi3:

total 1358

$\begin{array}{lc}\text {-rw-r--r-- } 1 \text { cj } & 156 \text { Nov } 9 \text { 15:42 desc.txt } \\ \text {-rw-r--r-- } 1 \text { cj } & 156 \text { Nov } 9 \text { 15:42 00index.txt } \\ \text {-rw-r--r-- } 1 \text { cj } & 7398 \text { Nov } 915: 42 \text { aletter.txt } \\ \text {-rw-r--r-- } 1 \text { cj } & 1886 \text { Nov } 915: 42 \text { copying } \\ \text {-rw-r--r-- } 1 \text { cj } & 81 \text { Nov } 915: 42 \text { diskoi3 } \\ \text {-rw-r--r-- } 1 \text { cj } & 15 \text { Nov } 915: 42 \text { install.end } \\ \text {-rw-r--r-- } 1 \text { cj } & 1366076 \text { Nov } 915: 46 \text { oistat.tgz }\end{array}$

pub/mirror/Linux/slackware/oop 1:

total 365

-rw-r--r-- 1 cj 130 Nov 9 15:46 desc.txt

-rw-r--r-- $1 \mathrm{cj} \quad 130$ Nov 9 15:46 00index.txt

-rw-r-r-- 1 cj 96 Nov 9 15:46 diskoop1

-rW-r--r-- $1 \mathrm{cj} \quad 33$ Nov $915: 46$ install.end

-rw-r--r-- 1 cj 354142 Nov 9 15:47 smaltalk.tgz

-rw-r--r-- 1 cj $\quad 44$ Nov $915: 47$ tagfile 
pub/mirror/Linux/slackware/q1:

total 648

\begin{tabular}{|c|c|}
\hline$-r w-r-r-r-1 c j$ & 208 Nov 9 15:47 desc.txt \\
\hline$-r w-r-r--1 c j$ & 208 Nov 9 15:47 00index.txt \\
\hline$-\mathrm{rw}-\mathrm{r}-\mathrm{-r}-\mathrm{l} 1 \mathrm{cj}$ & 3906 Nov 9 15:47 bootkem.log \\
\hline -rw-r--r-- $1 \mathrm{cj}$ & 1272 Nov 9 15:47 diskq1 \\
\hline$-r w-r--r--1 c j$ & 2950 Nov 9 15:47 idekern.log \\
\hline$-r w-r-r-r-1 c j$ & 285412 Nov $915: 48$ idekern.tgz \\
\hline$-r w-r-r--1 c j$ & 143 Nov 9 15:48 readme \\
\hline$-r w-r-r-1-1 c j$ & 3850 Nov $915: 48$ scsikern.log \\
\hline$-\mathrm{rw}-\mathrm{r}-\mathrm{-r}-\mathrm{-} 1 \mathrm{cj}$ & 339933 Nov 9 15:49 scsikern.tgz \\
\hline
\end{tabular}

pub/mirror/Linux/slackware/q2:

total 980

-rw-r--r-- 1 cj 104 Nov 9 15:49 .desc.txt

-rW-r--r-- 1 cj 104 Nov 9 15:49 00index.txt

-rw-r--r-- 1 cj 349 Nov 9 15:49 diskq2

-rW-r--r-- $1 \mathrm{cj} \quad 31$ Nov $915: 49$ install.end

-rw-r--r-- 1 cj 983098 Nov 9 15:52 lx99p13p.tgz

pub/mirror/Linux/slackware/scripts:

total 76

-rw-r--r-- 1 cj 156 Nov 9 15:52 desc.txt

-rw-r--r-- $1 \mathrm{cj} \quad 156$ Nov 9 15:52 00index.txt

-rw-r-r-- 1 cj 270 Nov 9 15:52 INDEX

-rW-r-r-- 1 cj 2519 Nov 9 15:52 makeboot 
-rw-r-r-- 1 cj 2674 Nov 9 15:52 makeflop

-rW-r-r-- 1 cj 19173 Nov 9 ij:52 pkgtool

-rw-r--r-- 1 cj 48619 Nov 9 15:52 setup

pub/mirror/Linux/slackware/tcll:

total 424

-rW-r-r-- 1 cj 156 Nov 9 15:52 .desc.txt

-rw-r--r-- 1 cj 156 Nov 9 15:52 00index.txt

-rw-r--r-- 1 cj 759 Nov 9 15:52 disktcll

-rW-r-r-- $1 \mathrm{cj} \quad 13$ Nov 9 15:52 install.end

-rW-r-r-- $1 \mathrm{cj} \quad 1630$ Nov $915: 52$ tagfile

$-r w-r--r-1$ cj 1630 Nov $915: 52$ tagfile.org

-rW-r--r-- 1 cj 416171 Nov $915: 54$ tcltkdis.tgz

pub/mirror/Linux/slackware/x 1 :

total 1366

-rw-r--r-- 1 cj 260 Nov 9 15:54 desc.txt

-rw-r-r-r- 1 cj 260 Nov 9 15:54 00index.txt

-rW-r-r-r- 1 cj 1138 Nov 9 15:54 diskx 1

-rw-r--r-- 1 cj $\quad 8896$ Nov 9 15:54 readme

-rW-r--r-- 1 cj 2550 Nov 9 15:54 tagfile

-rw-r-r-- $1 \mathrm{cj} \quad 2550$ Nov 9 15:54 tagfile.org

-rw-r-r-- $1 \mathrm{cj} \quad 2561$ Nov 9 15:54 tagfile.pat

-rw-r--r-- 1 cj 379570 Nov $915: 55$ x_8514.tgz

-rw-r-r-r- 1 cj 428066 Nov 9 15:57 x_mach32.tgz

-rW-r-r-r 1 cj 383529 Nov 9 15:58 x_mach8.tgz

-rW-r--r-- 1 cj 132831 Nov 9 15:58 xf_doc.tgz 
pub/mirror/Linux/slackware/x2:

total 1402

$\begin{array}{lc}\text {-rw-r-r-- } 1 \text { cj } & 182 \text { Nov } 915: 58 \text {.desc.txt } \\ \text {-rw-r-r-- } 1 \text { cj } & \text { 182 Nov } 915: 5800 \text { index.txt } \\ \text {-rw-r--r-- } 1 \text { cj } & 1272 \text { Nov } 915: 58 \text { diskx2 } \\ \text {-rw-r-r-- } 1 \text { cj } & 337989 \text { Nov } 915: 59 \text { x_mono.tgz } \\ \text {-rw-r-r-- } 1 \text { cj } & 417388 \text { Nov } 916: 00 \text { x_s3.tgz } \\ \text {-rw-r-r-- } 1 \text { cj } & 405704 \text { Nov } 916: 01 \text { x_svga.tgz } \\ \text {-rw-r--r-- } 1 \text { cj } & 63049 \text { Nov } 916: 01 \text { xf_cfg.tgz } \\ \text {-rw-r--r-- } 1 \text { cj } & 155813 \text { Nov } 916: 01 \text { xpm32g.tgz }\end{array}$

pub/mirror/Linux/slackware/x3:

total 1339

-rW-r-r-r- 1 cj 130 Nov 9 16:01 desc.txt

-rW-r-r-r $1 \mathrm{cj} \quad 130$ Nov 9 16:01 00index.txt

-rw-r--r-- 1 cj $\quad 577$ Nov 9 16:01 diskx3

-rw-r-r-r- 1 cj 357768 Nov $916: 02$ x_vga16.tgz

-rw-r--r-- $1 \mathrm{cj} \quad 685124$ Nov 9 16:04 xf_lib.tgz

-rW-r--r-- 1 cj 291072 Nov 9 16:04 xman1.tgz

pub/mirror/Linux/slackware/x4:

total 1395

-rW-r--r-- 1 cj 104 Nov 9 16:04 desc.txt

-rW-r-r-- 1 cj 104 Nov 9 16:04 00index.txt

-rW-r--r-- 1 cj 431 Nov 9 16:04 diskx4

-rW-r--r-- 1 cj 839711 Nov 9 16:07 xf_bin.tgz

-rW-r--r-- 1 cj 563200 Nov $916: 08$ xfonts1.tgz 
pub/mirror/Linux/slackware/x5:

total 1192

-rw-r--r-- 1 cj 130 Nov 9 16:08 desc.txt

-rW-r-r-r- $1 \mathrm{cj} \quad 130$ Nov 9 16:08 00index.txt

-rW-r-r-r 1 cj 436 Nov 9 16:08 diskx5

-rw-r-r-r- $1 \mathrm{cj} \quad 27$ Nov 9 16:08 install.end

-rw-r-r-- $1 \mathrm{cj} \quad 1179036$ Nov 9 16:10 xfonts2.tgz

-rW-r-r-- 1 cj 28128 Nov 9 16:10 xlock.tgz

pub/mirror/Linux/slackware/xap 1:

total 1334

-rW-r--r-- $1 \mathrm{cj} \quad 312$ Nov 9 16:11 desc.txt

-rw-r--r-- $1 \mathrm{cj} \quad 312$ Nov 9 16:11 00index.txt

-rw-r--r-- 1 cj 1264 Nov $916: 11$ diskxap 1

-rw-r-r-r- 1 cj 198531 Nov 9 16:11 gs_x11.tgz

-rw-r--r-- 1 cj 352123 Nov 9 16:12 libgr 13.tgz

-rw-r-r-r- 1 cj 133883 Nov $916: 12$ seyon.tgz

-rw-r-r-r $1 \mathrm{cj} \quad 746$ Nov 9 16:12 tagfile

-rW-r--r-- $1 \mathrm{cj} \quad 746$ Nov $916: 12$ tagfile.org

-rw-r--r-- $1 \mathrm{cj} \quad 747$ Nov 9 16:12 tagfile.pat

-rw-r--r-- 1 cj 14794 Nov 9 16:12 vgaset.tgz

-rw-r--r-- 1 cj 181826 Nov $916: 12$ workman.tgz

-rW-r-r-- 1 cj 192635 Nov 9 16:13 xfileman.tgz

-rW-r--r-- $1 \mathrm{cj} \quad 208506$ Nov $916: 13$ xv300.tgz

pub/mirror/Linux/slackware/xap2:

total 997

-rw-r--r-- 1 cj 156 Nov 9 16:13 desc.txt 
-rw-r--r-- 1 cj 156 Nov 9 16:1300index.txt

-rw-r--r-- $1 \mathrm{cj} \quad 581$ Nov 9 16:13 diskxap2

-rw-r--r-- 1 cj 328203 Nov $916: 14$ gchess.tgz

-rw-r-r-- $1 \mathrm{cj} \quad 33$ Nov 9 16:14 install.end

-rw-r--r-- 1 cj 82315 Nov $916: 14$ xfm12.tgz

-rw-r-r-- 1 cj 575837 Nov $916: 15$ xrest.tgz

pub/mirror/Linux/slackware/xd1:

total 1262

-rw-r--r-- 1 cj $\quad 182$ Nov 9 16:15 desc.txt

$-r w-r-r--1 \mathrm{cj} \quad 182$ Nov $916: 1500$ index.txt

-rw-r--r-- $1 \mathrm{cj} \quad 530$ Nov $916: 15$ diskxd1

-rw-r--r-- $1 \mathrm{cj} \quad 372$ Nov $916: 15$ tagfile

-rW-r--r-- $1 \mathrm{cj} \quad 372$ Nov $916: 16$ tagfile.org

-rw-r--r-- $1 \mathrm{cj} \quad 378$ Nov 9 16:16 tagfile.pat

-rw-r--r-- 1 cj 882894 Nov $916: 17$ xf_kit.tgz

-rw-r--r-- 1 cj 381219 Nov $916: 18$ xman3.tgz

pub/mirror/Linux/slackware/xd2:

total 1371

-rw-r-r-- $1 \mathrm{cj} \quad 104$ Nov 9 16:18 desc.txt

-rw-r--r-- $1 \mathrm{cj} \quad 104$ Nov 9 16:18 00index.txt

$-r W-r--r--1 c j \quad 492$ Nov $916: 18$ diskxd2

-rw-r--r-- $1 \mathrm{cj} \quad 785636$ Nov $916: 20 \mathrm{xf}$ _prog.tgz

-rw-r--r-- $1 \mathrm{cj} \quad 589852$ Nov $916: 21$ xkitlibl.tgz

pub/mirror/Linux/slackware/xd3: 
total 1081

$\begin{array}{lc}\text {-rw-r--r-- } 1 \mathrm{cj} & 130 \text { Nov } 916: 21 \text {.desc.txt } \\ \text {-rw-r--r-- } 1 \mathrm{cj} & 130 \text { Nov } 916: 2100 \text { index.txt } \\ \text {-rw-r--r-- } 1 \mathrm{cj} & 531 \text { Nov } 916: 21 \text { diskxd3 } \\ \text {-rw-r--r-- } 1 \mathrm{cj} & 10 \text { Nov } 916: 21 \text { install.end } \\ \text {-rw-r--r-- } 1 \mathrm{cj} & 1014600 \text { Nov } 916: 23 \text { xf_pex.tgz } \\ \text {-rw-r--r-- } 1 \mathrm{cj} & 77878 \text { Nov } 916: 23 \text { xkitlib2.tgz }\end{array}$

pub/mirror/Linux/slackware/xv1:

total 1446

\begin{tabular}{|c|c|}
\hline$-r w-r--r--1 c j$ & 208 Nov 9 16:23 .desc.txt \\
\hline$-r w-r--r--1 c j$ & 208 Nov 9 16:23 00index.txt \\
\hline$-r w-r--r--1 c j$ & 272 Nov 9 16:23 diskxvl \\
\hline$-r w-r-r--1 c j$ & 541 Nov $916: 23$ tagfile \\
\hline$-r w-r--r--1 c j$ & 541 Nov 9 16:23 tagfile.org \\
\hline$-r w-r--r--1 c j$ & 545 Nov $916: 24$ tagfile.pat \\
\hline$-r w-r--r--1 c j$ & 127112 Nov 9 16:24 xv32_sa.tgz \\
\hline$-r w-r--r--1 c j$ & 854927 Nov 9 16:26 xv32_so.tgz \\
\hline$-r w-r--r--1 c j$ & 453903 Nov $916: 26$ xvol32.tgz \\
\hline
\end{tabular}

pub/mirror/Linux/slackware/xv2:

total 1442

-rw-r--r-- $1 \mathrm{cj} \quad 182$ Nov 9 16:26 desc.txt

-rW-r--r-- $1 \mathrm{cj} \quad 182$ Nov 9 16:27 00index.txt

-rw-r--r-- $1 \mathrm{cj} \quad 486$ Nov $916: 27$ diskxv2

$-r w-r-r--1 \mathrm{cj} \quad 9$ Nov $916: 27$ install.end

-rw-r--r-- 1 cj 848510 Nov $916: 28$ xv32_a.tgz

-rW-r--r-- 1 cj 232996 Nov 9 16:29 xv32exmp.tgz

-rw-r--r-- 1 cj 346301 Nov 9 16:29 xvinc32.tgz

-rW-r--r-- 1 cj $\quad 5440$ Nov $916: 29$ xvmenus.tgz 
pub/mirror/Linux/slackware/y1:

total 968

\begin{tabular}{|c|c|}
\hline$-r w-r-r--1 c j$ & 156 Nov 9 16:29 .desc.txt \\
\hline$-r w-r-r--1 ~ c j$ & 156 Nov 9 16:29 00index.txt \\
\hline$-r W-r-r--1 c j$ & 972602 Nov $916: 31$ bsdgames.tgz \\
\hline$-r w-r-r--1 c j$ & 657 Nov 9 16:31 disky1 \\
\hline$-r w-r-r--1 c j$ & 23 Nov 9 16:31 install.end \\
\hline$-r w-r-r--1 c j$ & 1628 Nov 9 16:31 tagfile \\
\hline$-r W-r-r--1 c j$ & 1628 Nov 9 16:31 tagfile.org \\
\hline
\end{tabular}



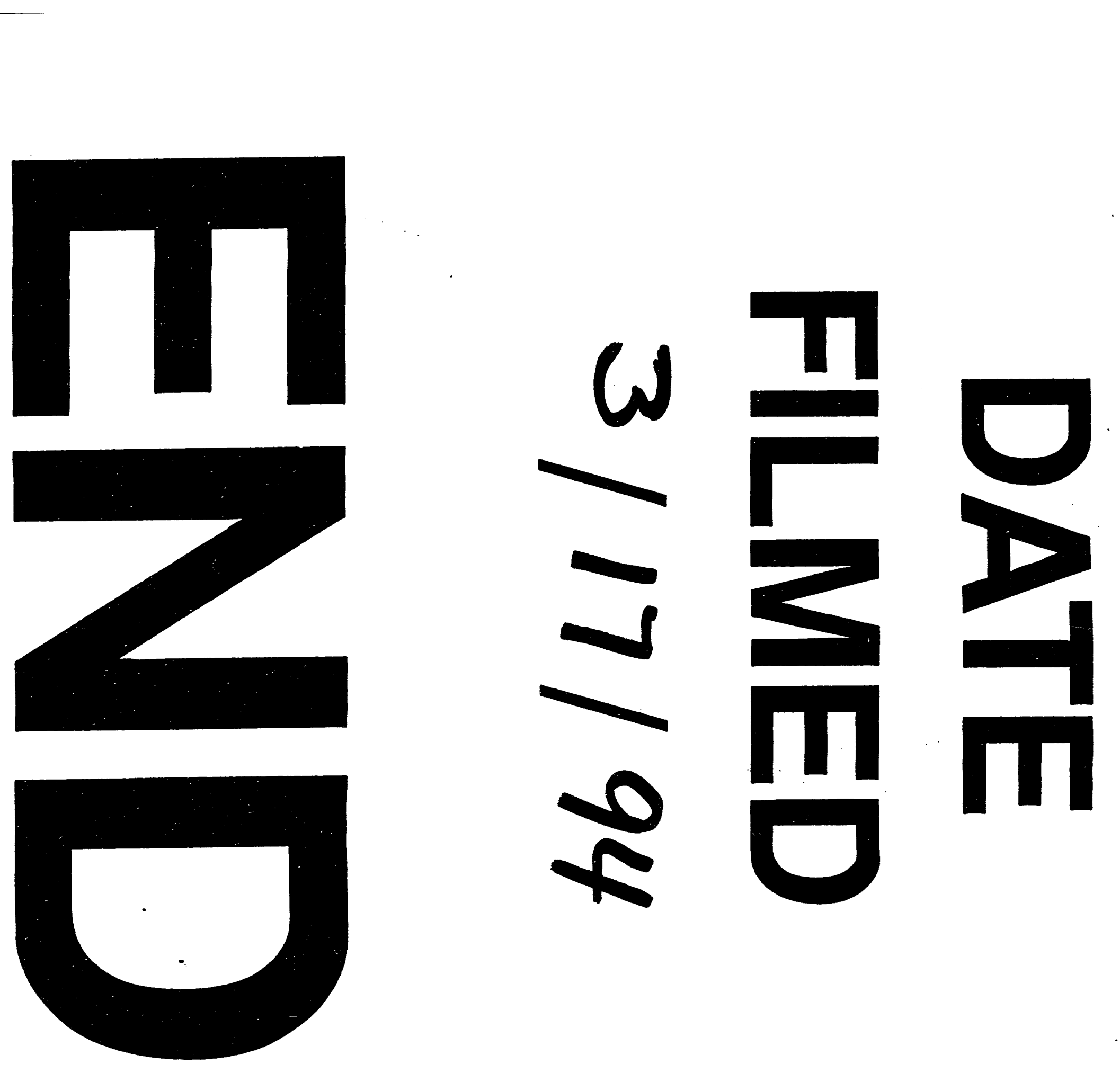
